\title{
One-point functions in massive integrable QFT with boundaries
}

\author{
Márton Kormos ${ }^{a, b}$ and Balázs Pozsgay ${ }^{c}$ \\ ${ }^{a}$ International School for Advanced Studies (SISSA), \\ Via Bonomea 265, 34136 Trieste, Italy \\ ${ }^{b}$ Istituto Nazionale di Fisica Nucleare, Sezione di Trieste, \\ Via Bonomea 265, 34136 Trieste, Italy \\ ${ }^{c}$ Institute for Theoretical Physics, Universiteit van Amsterdam, \\ Valckenierstraat 65, 1018 XE Amsterdam, The Netherlands \\ E-mail: kormos@sissa.it, pozsgay.balazs@gmail.com
}

ABSTRACT: We consider the expectation value of a local operator on a strip with non-trivial boundaries in 1+1 dimensional massive integrable QFT. Using finite volume regularisation in the crossed channel and extending the boundary state formalism to the finite volume case we give a series expansion for the one-point function in terms of the exact form factors of the theory. The truncated series is compared with the numerical results of the truncated conformal space approach in the scaling Lee-Yang model. We discuss the relevance of our results to quantum quench problems.

KeYwords: Boundary Quantum Field Theory, Exact S-Matrix, Integrable Field Theories, Bethe Ansatz

ARXIV EPRINT: 1002.2783 


\section{Contents}

1 Introduction 1

2 Boundary states and expectation values 3

$\begin{array}{lll}2.1 & \text { Boundary states in finite volume } & 7\end{array}$

$\begin{array}{lll}2.2 & \text { Large volume expansion of the Casimir energy } & 10\end{array}$

$\begin{array}{ll}2.3 & \text { Connection between boundary states and form factors } \\ \end{array}$

3 Evaluation of the vacuum expectation value $\quad 16$

$\begin{array}{lll}3.1 & \text { Evaluation of } D_{21} \text { and } D_{12} & 19\end{array}$

3.2 Evaluation of $D_{13}$ and $D_{31}$

3.3 Evaluation of $D_{22} \quad 22$

3.4 Connections to other problems 24

3.4.1 Comparison with a proposal of LeClair et al. 24

3.4.2 Relation to quench problems 25

3.4.3 Relation to finite temperature correlation functions 26

4 Comparison with TCSA $\quad 27$

4.1 The boundary scaling Lee-Yang model 27

4.1.1 The critical Lee-Yang model as a minimal conformal field theory 27

4.1.2 The scaling Lee-Yang model 30

$\begin{array}{ll}4.2 & \text { The comparison } \\ & 32\end{array}$

4.2.1 Expectation values in the truncated conformal space approach $\quad 32$

4.2.2 Comparing the form factor expansion with TCSA 33

5 Conclusions $\quad 35$

$\begin{array}{ll}\text { A Regularisation of the divergent sums } & 37\end{array}$

B Properties of the four-particle form factor 39

$\begin{array}{ll}\text { C Summary of our results } & 40\end{array}$

\section{Introduction}

Finite size effects play a central role in quantum field theory and statistical physics. Apart from having direct relevance to statistical physics models in finite volume, describing for example boundary critical phenomena or percolation problems, they naturally appear in the description of systems at finite temperature. For example, two-dimensional Euclidean field theories with a finite, periodic direction provide a framework for studying one-dimensional 
theories at finite temperature. Moreover, boundary phenomena and finite volume systems can play an important role in the understanding of quantum quenches: in certain cases the boundaries play the role of the initial and final states of the non-equilibrium problem [1-3]. In addition, in many cases, especially for numerical simulations, the system under consideration is put in a finite volume box. In this case it is essential to understand the finite size behaviour of various quantities.

Correlation functions are very important both at finite and infinite volume because they encode a lot of non-trivial information about the spectrum and the interactions in the theory. Calculating correlators is not a simple task even in integrable theories. In $1+1$ dimensional integrable quantum field theories the form factor approach provides an efficient way to calculate correlation functions at zero temperature and in infinite volume. The essence of the method is inserting a complete set of asymptotic states into the correlators and then making use of the explicit forms of the appearing matrix elements of the operators, the so-called form factors, which are known in many integrable models.

However, for finite temperature or equivalently, for finite geometry the applicability of the form factor approach at present is somewhat constrained. There has been considerable progress in free theories (for example the quantum Ising model), where the non-interacting nature of the theory allows for calculating correlation functions both in the finite temperature setting [4] or in the presence of a boundary [5, 6]. However, interacting theories pose technical and conceptual difficulties, which have not yet been overcome.

In the finite temperature case one particular approach was developed by LeClair and Mussardo [7]. They proposed an integral series for correlation functions based on the exact form factors and the Thermodynamic Bethe Ansatz equations. Their result for one-point functions were checked in particular examples [8-10], and then a highly non-trivial check was given by confirming it up to the third order in the low-temperature expansion using finite volume regularisation [11]. For two-point functions, however, some counterexamples were found where the formalism does not seem to work $[8,9]$ and the problem is still far from being settled.

In this work we address the generalised problem of one-point functions in finite volume where instead of periodic boundary conditions we consider non-trivial boundary conditions, thus we have a strip geometry instead of a cylinder. In this sense our work can be a first step in finding an expression similar to the LeClair-Mussardo formula in the boundary case. Expectation values in the presence of boundaries in general depend on the position of the operator in question. ${ }^{1}$ The exact determination of them is thus non-trivial; for one boundary the problem has comparable difficulty to that of the two-point functions in infinite volume; and similarly, in the presence of two boundaries the technical problems resemble the case of a three-point function or a two-point function at finite temperature.

In the work [13] the authors considered the case of one boundary, or in other words the problem when the operator is much closer to one of the boundaries than the volume of the system. They developed a form factor expansion which was later used in $[2,14]$

\footnotetext{
${ }^{1}$ This has to be contrasted with the problem of expectation values of boundary operators considered for example in [12].
} 
to study certain problems in condensed-matter systems. In addition, the analytic results were confronted in [13] with the numerical Truncated Conformal Space Approach (TCSA). Our expansion reproduces and goes beyond their result, and we will show that at small enough volume our results give a remarkable improvement. Our numerical comparison with the TCSA results is very similar to their method. On the other hand, the theoretical determination of the spectral series is more involved: there appear conceptual (and also technical) difficulties, which are not present in [13]. Most importantly, one has to deal with certain singularities of the form factors and also the divergent contributions to the partition function. In this work we make use of a finite volume regularisation scheme, which first was used in interacting field theory in $[11,15]$ and later in $[16,17]$.

As remarked above, integrable boundary field theory can be used to investigate quench problems. Recent papers $[2,3]$ consider the time evolution of one-point functions after certain types of global quenches. As we will show, their problem and method have similarities to ours and thus our results also have relevance to quench problems.

The outline of the paper is the following. In section 2 we introduce the finite volume boundary states and as a first application we give a series expansion of the finite volume ground state energy. In section 3 we turn to the problem of one-point functions. After describing the method in detail we present the explicit calculations of the first terms in the form factor expansion. At the end of the section we discuss the relation of our expression with existing results in the literature and we also explain its connection to quench problems. Section 4 is devoted to the numerical comparison between the spectral expansion and the TCSA data for the scaling Lee-Yang model, considered as a perturbed conformal field theory. First we collect the relevant properties and formulae of the model, then we discuss briefly the TCSA method and how one-point functions can be obtained in this framework. Finally we compare the form factor and TCSA results for various combinations of boundary conditions and strip widths. Our conclusions are given in section 5. For some technical details and for a collection of our final result the reader is referred to the appendices.

\section{Boundary states and expectation values}

For the sake of simplicity let us consider an integrable relativistic field theory with only one particle species with mass $m$ and two-particle S-matrix $S(\theta)$. We are interested in the case where the theory lives on a finite line segment of length $R$ with boundary conditions at the edges that do not spoil the integrability.

If $B$ denotes such an integrable boundary then the scattering of an incoming multiparticle state, ${ }^{2}$

$$
\left|A\left(\theta_{1}\right) A\left(\theta_{2}\right) \ldots A\left(\theta_{n}\right)\right\rangle_{B},
$$

is purely elastic, i.e. the set of outgoing rapidities is $\left\{-\theta_{1},-\theta_{2}, \ldots,-\theta_{N}\right\}$ and the scattering can be described by a product of one-particle reflection amplitudes which are defined as [18]

$$
|A(\theta)\rangle_{B}=R_{B}(\theta)|A(-\theta)\rangle_{B}
$$

\footnotetext{
${ }^{2}$ The subscript $B$ indicates that the state is in the Hilbert space of the boundary theory.
} 


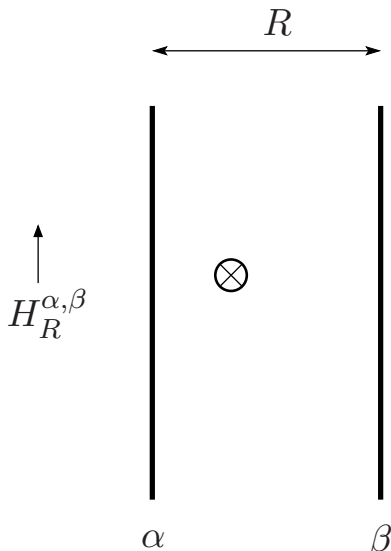

(a)

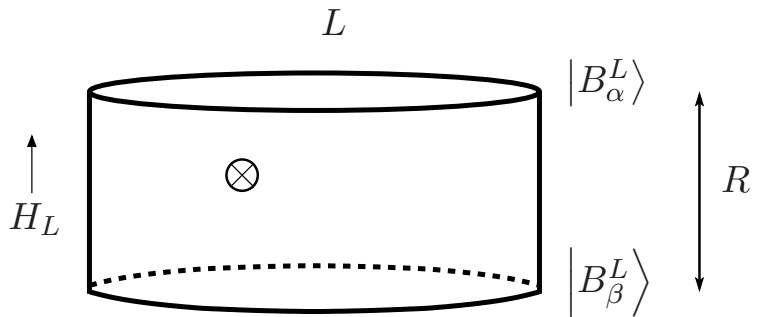

(b)

Figure 1. One-point function in the original strip geometry (a) and the finite volume regularisation performed in the crossed channel (b). The upward arrows denote the (imaginary) time evolution generated by the corresponding Hamiltonians.

The main objective of this paper is to evaluate the vacuum expectation value

$$
\langle O(x)\rangle_{R}^{\alpha, \beta}
$$

in the presence of the integrable boundaries $\alpha$ and $\beta$ where $x \in[0, R]$. Here the expectation value is taken with respect to the vacuum state of the finite volume Hamiltonian $H_{R}^{\alpha, \beta}$ (see figure 1(a)). In a more general setting one can also take excited states corresponding to boundary bound states but we do not elaborate on this case here.

One can consider the same quantity (2.3) after a Euclidean rotation. In this picture $R$ plays the role of the Euclidean time variable and the expectation value is given by the formal expression

$$
\langle O(x)\rangle_{R}^{\alpha, \beta}=\frac{\left\langle B_{\alpha}\left|e^{-H x} O e^{-H(R-x)}\right| B_{\beta}\right\rangle}{\left\langle B_{\alpha}\left|e^{-H R}\right| B_{\beta}\right\rangle},
$$

where $H$ is the infinite volume Hamiltonian and $\left|B_{\alpha}\right\rangle$ and $\left|B_{\beta}\right\rangle$ are the boundary states corresponding to the boundary conditions $\alpha$ and $\beta$, respectively. When they do not contain zero-momentum particles, they can be expanded in the asymptotic multi-particle basis as [18]

$$
\left|B_{j}\right\rangle=\mathcal{N}_{j} \exp \left(\int \frac{d \theta}{4 \pi} K_{j}(\theta) A(-\theta) A(\theta)\right)|0\rangle,
$$

where

$$
K_{j}(\theta)=R_{j}(i \pi / 2-\theta), \quad j=\alpha, \beta
$$

and $A(\theta)$ are the Faddeev-Zamolodchikov creation operators satisfying the commutation relations

$$
A\left(\theta_{1}\right) A\left(\theta_{2}\right)=S\left(\theta_{1}-\theta_{2}\right) A\left(\theta_{2}\right) A\left(\theta_{1}\right) .
$$

The integrals run from $-\infty$ to $\infty$ unless otherwise stated. The amplitudes $K_{i}(\theta)$ satisfy the "boundary cross-unitary equation" [18]

$$
K_{j}(\theta)=S(2 \theta) K_{j}(-\theta),
$$


which serves as a consistency relation of (2.5). The behaviour under complex conjugation is given by $K_{j}(\theta)^{*}=K_{j}(-\theta)$.

In general there may be additional contributions to the boundary state involving zeromomentum particles, which can be associated with a non-zero coupling of a single particle state to the boundary in the original channel. In this case there is a corresponding pole in the reflection factor at $\theta=i \pi / 2$ :

$$
R_{j}(\theta) \sim \frac{i}{2} \frac{\left(g_{j}\right)^{2}}{\theta-i \pi / 2}
$$

and the boundary state is given by

$$
\left|B_{j}\right\rangle=\mathcal{N}_{j} \exp \left(\bar{g}_{j} A(0)+\int \frac{d \theta}{4 \pi} K_{j}(\theta) A(-\theta) A(\theta)\right)|0\rangle,
$$

where $\bar{g}_{j}$ represents the one-particle coupling to the boundary. Originally it was argued that $\bar{g}_{j}=g_{j}[18]$, however it was found numerically in [13] that the proper normalisation is $\overline{g_{j}}=g_{j} / 2$. This claim was later proven on general grounds in $[19,20]$. The normalisation constants $\mathcal{N}_{j}$ are infinite in the infinite volume system. However, this is not a serious problem as they drop out from the calculation of the vacuum expectation values and one may set them to unity.

The general strategy to evaluate (2.4) now is the following. We substitute expression (2.6) for the boundary states and expand the exponentials. The resulting multi-particle states are eigenstates of the Hamiltonian, and the matrix elements of the local operator $O$ between these states are the form factors, which can be determined in principle and which are actually known in many integrable models.

The special case for the $R \rightarrow \infty$ limit of (2.4) with $x$ fixed (one-point function in the presence of only one boundary) was investigated in [13]. In this case there are no conceptual and technical difficulties and the spectral expansion described above (valid for $\left.g_{\alpha}=0\right)$ reads

$$
\langle O(x)\rangle=\sum_{n=0}^{\infty} \frac{1}{2^{n} n !} \int \frac{d \theta_{1}}{2 \pi} \ldots \frac{d \theta_{n}}{2 \pi} F_{2 n}^{O}\left(-\theta_{1}, \theta_{1}, \ldots,-\theta_{n}, \theta_{n}\right) \prod_{i=1}^{n}\left(K_{\alpha}\left(\theta_{i}\right) e^{-2 m \cosh \theta_{i} x}\right),
$$

where

$$
F_{m}^{O}\left(\theta_{1}, \ldots, \theta_{m}\right)=\left\langle 0|\mathcal{O}| \theta_{1}, \ldots, \theta_{m}\right\rangle
$$

are the elementary form factors of $O$ in infinite volume. In integrable models they are meromorphic functions of the rapidities which satisfy the so-called form factor axioms (Watson equations), which can be considered as axioms for the form factor bootstrap. Supplied with the principles of maximum analyticity and the cluster property they contain enough information to determine the form factors completely. In a theory with only one particle species the form factor axioms read:

I. Lorentz invariance:

$$
F_{n}^{O}\left(\theta_{1}+\Lambda, \ldots, \theta_{n}+\Lambda\right)=e^{s \Lambda} F_{n}^{O}\left(\theta_{1}, \ldots, \theta_{n}\right)
$$


where $s$ is the Lorentz spin of the operator $O$. In this work we only consider scalar operators corresponding to $s=0$.

II. Exchange:

$$
F_{n}^{O}\left(\theta_{1}, \ldots, \theta_{k}, \theta_{k+1}, \ldots, \theta_{n}\right)=S\left(\theta_{k}-\theta_{k+1}\right) F_{n}^{O}\left(\theta_{1}, \ldots, \theta_{k+1}, \theta_{k}, \ldots, \theta_{n}\right) .
$$

III. Cyclic permutation:

$$
F_{n}^{O}\left(\theta_{1}+2 i \pi, \theta_{2}, \ldots, \theta_{n}\right)=F_{n}^{O}\left(\theta_{2}, \ldots, \theta_{n}, \theta_{1}\right) .
$$

IV. Kinematical singularity

$$
-i \underset{\theta=\theta^{\prime}}{\operatorname{Res}} F_{n+2}^{O}\left(\theta+i \pi, \theta^{\prime}, \theta_{1}, \ldots, \theta_{n}\right)=\left(1-\prod_{k=1}^{n} S\left(\theta-\theta_{k}\right)\right) F_{n}^{O}\left(\theta_{1}, \ldots, \theta_{n}\right) .
$$

V. Dynamical singularity

$$
-i \operatorname{Res}_{\theta=\theta^{\prime}} F_{n+2}^{\mathcal{O}}\left(\theta+i u, \theta^{\prime}-i u, \theta_{1}, \ldots, \theta_{n}\right)=\Gamma F_{n+1}^{\mathcal{O}}\left(\theta, \theta_{1}, \ldots, \theta_{n}\right),
$$

where $\Gamma$ is the on-shell three-particle coupling corresponding to a bound state pole of the S-matrix

$$
S(\theta \sim i 2 u) \sim \frac{i \Gamma^{2}}{\theta-i 2 u}, \quad \theta \sim 2 i u .
$$

In a theory with only one particle, like the scaling Lee-Yang model the only possibility is $u=\pi / 3$.

We also note that all form factors can be expressed in terms of the elementary form factors with the help of the crossing relation

$$
\begin{aligned}
& F_{m n}^{O}\left(\theta_{1}^{\prime}, \ldots, \theta_{m}^{\prime} \mid \theta_{1}, \ldots, \theta_{n}\right)=F_{m-1, n+1}^{O}\left(\theta_{1}^{\prime}, \ldots, \theta_{m-1}^{\prime} \mid \theta_{m}^{\prime}+i \pi, \theta_{1}, \ldots, \theta_{n}\right) \\
& \quad+\sum_{k=1}^{n}\left(2 \pi \delta\left(\theta_{m}^{\prime}-\theta_{k}\right) \prod_{l=1}^{k-1} S\left(\theta_{l}-\theta_{k}\right) \times F_{m-1, n-1}^{O}\left(\theta_{1}^{\prime}, \ldots, \theta_{m-1}^{\prime} \mid \theta_{1}, \ldots, \theta_{k-1}, \theta_{k+1} \ldots, \theta_{n}\right)\right) .
\end{aligned}
$$

For an introduction to the form factor bootstrap program we refer the reader to the review of Smirnov [21] and to papers [22-25].

In the simultaneous presence of the two boundaries the expression (2.4) is ill-defined. There are divergent contributions both to the numerator and the denominator, which are associated with disconnected terms of the form factors and with various contributions to the partition function. One way to evaluate (2.4) is to introduce finite volume as a regulator of the singular contributions. This method was successfully used in [11] to derive a rigorous low-temperature expansion for one-point functions at finite temperature, which in the crossed channel corresponds to vacuum expectation values in a finite volume with periodic boundary conditions. In this sense the present work is an extension of the approach of [11] to the more general setting of arbitrary (integrable) boundary conditions. 
We introduce a large finite volume $L$ in the crossed channel (with periodic boundary conditions) and consider the limit

$$
\langle O(x)\rangle_{R}=\lim _{L \rightarrow \infty}\langle O(x)\rangle_{R}^{L}=\lim _{L \rightarrow \infty} \frac{\left\langle B_{\alpha}^{L}\left|e^{-H_{L} x} O(0,0) e^{-H_{L}(R-x)}\right| B_{\beta}^{L}\right\rangle}{\left\langle B_{\alpha}^{L}\left|e^{-H_{L} R}\right| B_{\beta}^{L}\right\rangle},
$$

where $H_{L}$ is the finite volume Hamiltonian and $\left|B_{j}^{L}\right\rangle$ represent the boundary states in finite volume (see figure 1(b)). It will be the subject of the next subsection to properly expand them in the basis of the eigenstates of $H_{L}$.

\subsection{Boundary states in finite volume}

The boundary states can be expanded in finite volume as

$$
\left|B_{j}^{L}\right\rangle=\sum_{\Psi} G_{j}^{\Psi}(L)|\Psi\rangle_{L}, \quad j=\alpha, \beta
$$

where $|\Psi\rangle_{L}$ are eigenstates of the finite volume Hamiltonian $H_{L}$. In particular the function $G_{j}^{0}(L)$ determines the large $R$ behaviour of the partition function and can be written as

$$
G_{j}^{0}(L)=e^{-f_{j} L} g_{j}^{0}(L)
$$

Here $f_{j}$ is the contribution of a single boundary to the ground state energy $E_{0}(R)$ and $g_{j}^{0}(L)$ is the standard non-perturbative $g$-function ${ }^{3}$ which was introduced in critical systems by Affleck and Ludwig [26]. In theories with only massive excitations in the bulk the $g$-function decays exponentially. The exact integral series for the $g$-function in massive theories was derived in [27]; for a first treatment of a non-trivial massless flow see [28].

The excited states $|\Psi\rangle_{L}$ of a finite volume Hamiltonian can be described in a large volume as scattering states consisting of $n$ particles with rapidities $\theta_{n}$ given by the solution of the asymptotic Bethe-Yang equations

$$
Q_{k}=m L \sinh \theta_{k}+\sum_{j \neq k} \delta\left(\theta_{k}-\theta_{j}\right)=2 \pi I_{k}, \quad k=1, \ldots, n
$$

It is convenient to define a continuous two-particle phase shift function by ${ }^{4}$

$$
S(\theta)=-e^{i \delta(\theta)}, \quad \delta(-\theta)=-\delta(\theta) .
$$

This poses the following prescription for the momentum quantum numbers:

$$
I_{k} \in \mathbb{Z} \quad \text { for odd } n, \quad I_{k} \in \mathbb{Z}+\frac{1}{2} \quad \text { for even } n .
$$

\footnotetext{
${ }^{3}$ This should not be confused with the one-particle boundary coupling $g_{j}$.

${ }^{4}$ We made use of the fact that the effective statistics is fermionic, i.e. $S(0)=-1$. All known integrable models possess this property, the only counter-example being the free boson. In a theory with more than one particle species the most convenient choice for the definition of the phase shift is $S_{a b}(\theta)=S_{a b}(0) e^{i \delta_{a b}(\theta)}$.
} 
The quantum numbers $\left\{I_{1}, \ldots, I_{n}\right\}$ completely determine the individual scattering states, therefore they may be used to label the sates as

$$
\left|\left\{I_{1} \ldots I_{n}\right\}\right\rangle_{L}
$$

where the states are normalised to unity:

$$
{ }_{L}\left\langle\left\{I_{1} \ldots I_{n}\right\} \mid\left\{I_{1}^{\prime} \ldots I_{m}^{\prime}\right\}\right\rangle_{L}=\delta_{n m} \delta_{I_{1}, I_{1}^{\prime}} \ldots \delta_{I_{n}, I_{n}^{\prime}} .
$$

The total energy and momentum are calculated additively as

$$
E=\sum_{i=1}^{n} m \cosh \theta_{i}+\mathcal{O}\left(e^{-\mu L}\right), \quad P=\sum_{i=1}^{n} m \sinh \theta_{i}+\mathcal{O}\left(e^{-\mu L}\right) .
$$

The exponential corrections are governed by the mass scale $\mu$ which is uniform in the sense that it is determined by the analytic structure of the S-matrix and it does not depend on the particular multi-particle state in question. For a systematic treatment of exponential corrections to excitation energies see $[29,30]$ and also recent papers [31, 32]. In a massive field theory it is expected that the leading exponential corrections of more complicated quantities (like form factors [33] and correlation functions) are also of order $e^{-\mu L}$.

For later use we introduce the density of states (in rapidity-space) in the $n$-particle sector of the theory, which is given by the Jacobian

$$
\rho_{n}\left(\theta_{1}, \ldots, \theta_{n}\right)=\operatorname{det} J_{k l}, \quad J_{k l}=\frac{\partial Q_{k}}{\partial \theta_{l}} .
$$

Apart from normalisation issues the boundary states in finite and infinite volume should have the same structure. Therefore, it is natural to expand the finite volume boundary state up to the two-particle contribution as

$$
\left|B_{j}^{L}\right\rangle=G_{j}^{0}(L)\left(|0\rangle_{L}+\frac{g_{i}}{2} N_{1}(L)|\{0\}\rangle_{L}+\sum_{I>0} K_{j}(\theta) N_{2}(\theta, L)|\{-I, I\}\rangle_{L}+\ldots\right),
$$

where in the last term it is understood that the rapidities $\theta$ are the solutions of the appropriate Bethe-Yang equation

$$
\bar{Q}_{1}(\theta)=m L \sinh \theta+\delta(2 \theta)=2 \pi I .
$$

Note also that the sum in (2.17) only runs over $I>0$ because the states $|\{I,-I\}\rangle_{L}$ and $|\{-I, I\}\rangle_{L}$ are identical. In (2.17) we introduced the normalisation factors $N_{1}(L)$ and $N_{2}(\theta, L)$ which are not determined by first principles. In the following we calculate them including all finite size effects which scale as negative powers of $L$ (only neglecting those which decay exponentially). To this order we consider vacuum expectation values in the $R \rightarrow \infty$ limit with fixed $x$, both in a large finite volume $L$ and directly in the infinite system.

In infinite volume the first three contributions read [13]

$$
\langle O(x)\rangle_{\alpha}=\langle O\rangle+\frac{g_{\alpha}}{2} F_{1}^{O} e^{-m x}+\frac{1}{2} \int \frac{d \theta}{2 \pi} K_{\alpha}^{*}(\theta) F_{2}^{O}(\theta,-\theta) e^{-2 m x \cosh \theta}+\ldots .
$$


The same quantity can also be evaluated in finite volume as

$$
\langle O(x)\rangle_{\alpha}^{L}=\langle O\rangle+\frac{g_{\alpha}}{2} N_{1}(L)\langle\{0\}|O| 0\rangle_{L} e^{-E_{0} x}+\sum_{I>0} K_{\alpha}^{*}(\theta) N_{2}(\theta, L)\langle\{-I, I\}|O| 0\rangle_{L} e^{-E_{I} x}+\ldots,
$$

where

$$
E_{0}=m+\mathcal{O}\left(e^{-\mu L}\right), \quad E_{I}=2 m \cosh \theta+\mathcal{O}\left(e^{-\mu L}\right),
$$

and the finite volume form factors are given by [15]

$$
\langle\{0\}|O| 0\rangle_{L}=\frac{F_{1}^{O}}{\sqrt{m L}}+\mathcal{O}\left(e^{-\mu L}\right), \quad\langle\{-I, I\}|O| 0\rangle_{L}=\frac{F_{2}^{O}(\theta,-\theta)}{\sqrt{\rho_{2}(\theta,-\theta)}}+\mathcal{O}\left(e^{-\mu L}\right) .
$$

The summation in (2.20) can be replaced by an integration leading to

$$
\begin{aligned}
\langle O(x)\rangle_{\alpha}^{L}= & \langle O\rangle+\frac{g_{\alpha}}{2} \frac{N_{1}(L)}{\sqrt{m L}} F_{1}^{O} e^{-m x}+ \\
& +\frac{1}{2} \int \frac{d \theta}{2 \pi} \frac{\bar{\rho}_{1}(\theta) N_{2}(\theta, L)}{\sqrt{\rho_{2}(\theta,-\theta)}} K_{\alpha}^{*}(\theta) F_{2}^{O}(\theta,-\theta) e^{-2 m x \cosh \theta}+\mathcal{O}\left(e^{-3 m x}\right)+\mathcal{O}\left(e^{-\mu L}\right),
\end{aligned}
$$

where $\bar{\rho}_{1}(\theta)$ is the constrained density of states given by

$$
\bar{\rho}_{1}(\theta)=\frac{d \bar{Q}_{1}}{d \theta}=m L \cosh \theta+2 \varphi(2 \theta)
$$

In a massive theory

$$
\langle O(x)\rangle_{\alpha}-\langle O(x)\rangle_{\alpha}^{L} \sim \mathcal{O}\left(e^{-\mu L}\right) .
$$

Requiring that expressions (2.19) and (2.21) only differ by exponentially small terms for any scalar operator yields

$$
N_{1}(L)=\sqrt{m L}+\mathcal{O}\left(e^{-\mu L}\right), \quad N_{2}(\theta, L)=\frac{\sqrt{\rho_{2}(\theta,-\theta)}}{\bar{\rho}_{1}(\theta)}+\mathcal{O}\left(e^{-\mu L}\right) .
$$

Note that this argument essentially coincides with that used in [15] to determine the normalisation factors of the elementary finite volume form factors.

The two-particle density satisfies

$$
\rho_{2}(\theta,-\theta)=\rho_{1}(\theta) \bar{\rho}_{1}(\theta)
$$

therefore

$$
N_{2}(\theta, L)=\sqrt{\frac{\rho_{1}(\theta)}{\bar{\rho}_{1}(\theta)}}=1-\frac{\varphi(2 \theta)}{m L \cosh \theta}+\mathcal{O}\left(1 / L^{2}\right) .
$$

We stress that the eqs. (2.22) include all finite volume corrections which behave as negative powers of $L$, in particular there can be no additional $\mathcal{O}(1 / L)$ corrections to $N_{2}$. This will become important in the evaluation of vacuum expectation values at finite $R$. 
It is straightforward to extend the argument above to higher excited states. The finite volume boundary state is given up to the four-particle terms by

$$
\begin{aligned}
\left|B_{j}^{L}\right\rangle= & G_{j}^{0}(L)\left(|0\rangle_{L}+\frac{g_{j}}{2} \sqrt{m L}|\{0\}\rangle_{L}+\sum_{I} K_{j}(\theta) N_{2}(\theta, L)|\{I,-I\}\rangle_{L}\right. \\
& \left.+\sum_{I} \frac{g_{j}}{2} K_{j}(\theta) N_{3}(\theta, L)|\{I,-I, 0\}\rangle_{L}+\frac{1}{2} \sum_{\substack{I J \\
I \neq J}} K_{j}\left(\theta_{1}\right) K_{j}\left(\theta_{2}\right) N_{4}\left(\theta_{1}, \theta_{2}, L\right)|\{I,-I, J,-J\}\rangle_{L}\right) .
\end{aligned}
$$

The three-particle sector consists of states with rapidities $\{-\theta, 0, \theta\}$ where $\theta$ is determined by the single quantisation condition

$$
\bar{Q}_{3}(\theta)=m L \sinh \theta+\delta(\theta)+\delta(2 \theta)=2 \pi I .
$$

Therefore the normalisation of the three-particle states is given by

$$
N_{3}(\theta)=\frac{\sqrt{\rho_{3}(\theta, 0,-\theta)}}{\bar{\rho}_{3}(\theta)},
$$

where

$$
\bar{\rho}_{3}(\theta)=\frac{d \bar{Q}_{3}(\theta)}{d \theta}=m L \cosh \theta+\varphi(\theta)+2 \varphi(2 \theta) .
$$

In the four-particle case the quantisation condition for the rapidities $\left\{-\theta_{1}, \theta_{1},-\theta_{2}, \theta_{2}\right\}$ is given by the system of equations

$$
\begin{aligned}
& \bar{Q}_{4,1}=m L \sinh \theta_{1}+\delta\left(\theta_{1}-\theta_{2}\right)+\delta\left(\theta_{1}+\theta_{2}\right)+\delta\left(2 \theta_{1}\right)=2 \pi I_{1}, \\
& \bar{Q}_{4,2}=m L \sinh \theta_{2}+\delta\left(\theta_{2}-\theta_{1}\right)+\delta\left(\theta_{1}+\theta_{2}\right)+\delta\left(2 \theta_{2}\right)=2 \pi I_{2},
\end{aligned}
$$

yielding the normalisation

$$
N_{4}\left(\theta_{1}, \theta_{2}\right)=\frac{\sqrt{\rho_{4}\left(\theta_{1},-\theta_{1}, \theta_{2},-\theta_{2}\right)}}{\bar{\rho}_{4}\left(\theta_{1}, \theta_{2}\right)},
$$

where

$$
\bar{\rho}_{4}\left(\theta_{1}, \theta_{2}\right)=\operatorname{det} J \quad \text { with } \quad J_{i k}=\frac{\partial \bar{Q}_{4, i}}{\partial \theta_{k}}, \quad i, k=1,2 .
$$

\subsection{Large volume expansion of the Casimir energy}

Before turning to the evaluation of the one-point function we consider the Casimir energy in the two-boundary setting. In [34] the exact non-perturbative value was found to be

$$
E_{0}(R)=-\frac{1}{2} \int \frac{d \theta}{2 \pi} m \cosh \theta \log \left(1+K_{\alpha}^{*}(\theta) K_{\beta}(\theta) e^{-\varepsilon(\theta)}\right),
$$

where $\varepsilon(\theta)$ is the solution of the boundary TBA equation

$$
\varepsilon(\theta)=2 m R \cosh \theta-\int \frac{d \theta}{2 \pi} \varphi\left(\theta-\theta^{\prime}\right) \log \left(1+K_{\alpha}^{*}\left(\theta^{\prime}\right) K_{\beta}\left(\theta^{\prime}\right) e^{-\varepsilon\left(\theta^{\prime}\right)}\right),
$$


where $\varphi(\theta)=\mathrm{d} \delta(\theta) / \mathrm{d} \theta$. Expression (2.26) is normalised to have the asymptotics $E_{0}(R) \rightarrow 0$ as $R \rightarrow \infty$. The actual ground state energy also includes bulk and boundary contributions, which can be calculated from the boundary TBA [35].

Equations (2.26)-(2.27) were derived in [34] for the case $g_{\alpha}=g_{\beta}=0$ but it was argued that they yield the correct result even in the presence of zero-momentum particles. Although this claim was supported by several numerical checks [35], a rigorous proof is still missing. Moreover, it turned out that there are certain cases when (2.26)-(2.27) cannot be true.

In $[19,20,36]$ it was shown that if $g_{\alpha} \neq 0 \neq g_{\beta}$ then the leading contribution in the integral in (2.26) is given by

$$
E_{0}(R)=-m \frac{\left|g_{\alpha} g_{\beta}\right|}{4} e^{-m R}+\ldots
$$

On the other hand, the authors argued that the leading behaviour of the Casimir energy is always

$$
E_{0}(R)=-m \frac{g_{\alpha} g_{\beta}}{4} e^{-m R}+\ldots,
$$

irrespective of the sign of $g_{\alpha} g_{\beta}$. This discrepancy shows that the validity of $(2.26)$ is restricted to the case $g_{\alpha} g_{\beta}>0$. On the other hand, it was shown in $[19,20,36]$ that it is possible to derive a proper analytic continuation to the region $g_{\alpha} g_{\beta}<0$ with the correct leading behaviour (2.29).

Here we derive a large $R$ expansion of the Casimir energy by means of the finite volume regularisation of the partition function. Our results will be compared to (2.26). We follow closely the treatment of $[19,20]$, however we are able to extend their method to obtain the second order terms of the Casimir energy. In order to keep the exposition simple, we only consider the region $g_{\alpha} g_{\beta}>0$ where the BTBA (2.26)-(2.27) is applicable.

The partition function $Z(L, R)$ can be evaluated in two different channels. Treating $L$ as time variable and $R$ as space one obtains in the large $L$ limit

$$
Z=e^{-E_{0}(R) L}\left(1+\mathcal{O}\left(e^{-m L}\right)\right),
$$

where $E_{0}(R)$ is the ground-state energy in a finite volume $R$. It follows that

$$
E_{0}(R)=-\lim _{L \rightarrow \infty} \frac{\log Z}{L}
$$

Treating $R$ as time variable (corresponding to thermal field theory with temperature $T=$ $1 / R)$ the partition function can be developed into a low-temperature expansion. In the regime $m L e^{-m R} \ll 1$ it is sufficient to sum over the low-lying excitations:

$$
Z=1+N_{1}^{2} \frac{g_{\alpha} g_{\beta}}{4} e^{-m R}+\sum_{I}\left(N_{2}(\theta)\right)^{2} K_{\alpha}^{*}(\theta) K_{\beta}(\theta) e^{-2 m R \cosh \theta}+\mathcal{O}\left(e^{-3 m R}\right) .
$$

Note that in the expression above we did not perform a complex conjugation for $g_{\alpha}$, although the amplitude $K_{\alpha}(\theta)$ is conjugated. The motivation for this prescription is a delicate issue. In unitary theories the one-particle couplings are always real, therefore it 
is only the non-unitary case which has to be addressed. In non-unitary theories $g_{\alpha}$ is purely imaginary, therefore complex conjugation simply results in changing the sign of the appropriate term. On the other hand, the space of the states is not positive-definite, in fact one-particle states can be assigned a negative norm. This additional change of sign accounts for the correct prescription in (2.30). A different argument is provided by the corresponding conventions in CFT, where the prescription for the inner product does not include complex conjugation. Moreover, when comparing our form factor expansion with TCSA results in section 4.2 we will show that this choice of the sign is the correct one.

If $m L \gg 1$ one can safely substitute the normalisations (2.22) into (2.30). The logarithm of the partition function is then written as

$$
\begin{aligned}
& \frac{\log Z}{L}=m \frac{g_{\alpha} g_{\beta}}{4} e^{-m R}+\sum_{I} \frac{m \cosh \theta}{\bar{\rho}_{1}(\theta)} K_{\alpha}^{*}(\theta) K_{\beta}(\theta) e^{-2 m R \cosh \theta} \\
& \quad-\frac{1}{2} m^{2} L\left(\frac{g_{\alpha} g_{\beta}}{4} e^{-m R}\right)^{2}+\mathcal{O}\left(e^{-\mu L}\right)+\mathcal{O}\left(e^{-3 m R}\right) .
\end{aligned}
$$

The first term is already in complete accordance with (2.29); in the following we also evaluate the second order terms. First of all note that the summation over $I$ is divergent because of the double pole

$$
K_{\alpha}^{*}(\theta) K_{\beta}(\theta) \approx \frac{g_{\alpha}^{2} g_{\beta}^{2}}{4} \frac{1}{\theta^{2}}, \quad \theta \approx 0 .
$$

In order to determine the leading singularity it suffices to use the lowest order approximation in (2.15)

$$
\theta_{I}=\frac{2 \pi I}{m L}, \quad I=\frac{1}{2}, \frac{3}{2}, \frac{5}{2}, \ldots
$$

The divergent part of the summation in (2.31) can then be expressed as

$$
e^{-2 m R} \sum_{I} \frac{g_{\alpha}^{2} g_{\beta}^{2}}{4 L}\left(\frac{m L}{2 \pi I}\right)^{2}=m^{2} L e^{-2 m R} \frac{g_{\alpha}^{2} g_{\beta}^{2}}{32},
$$

where we used the identity

$$
\left(\frac{1}{2}\right)^{2}+\left(\frac{3}{2}\right)^{2}+\left(\frac{5}{2}\right)^{2}+\cdots=\frac{\pi^{2}}{2}
$$

Therefore the $O\left(L e^{-2 m R}\right)$ terms cancel exactly in (2.31), as needed to obtain a meaningful $L \rightarrow \infty$ limit. Note that this result provides new evidence for the normalisation condition $\bar{g}_{j}=g_{j} / 2$, because the $L \rightarrow \infty$ limit would not exist with a different choice of $\bar{g}_{j}$.

In the following we also evaluate the finite left-over piece of the $\mathcal{O}\left(e^{-2 m R}\right)$ terms in (2.31). In order to make calculations as transparent as possible we first introduce the following theorem:

Theorem 1 Let $\theta_{I}$ be the solutions of the quantisation condition

$$
\bar{Q}_{1}(\theta)=m L \sinh \theta+\delta(2 \theta)=2 \pi I
$$


and let $f(\theta)$ be a symmetric function which apart from a double pole at $\theta=0$ is analytic in a neighbourhood of the real axis:

$$
f(\theta) \approx \frac{G}{\theta^{2}} \quad \text { as } \quad \theta \rightarrow 0 .
$$

Then the expression

$$
S(L)=\left(\sum_{I} \frac{f\left(\theta_{I}\right)}{\bar{\rho}_{1}\left(\theta_{I}\right)}\right)-\frac{G}{8} m L
$$

has a regular behaviour at large $L$ with the $L \rightarrow \infty$ limit given by

$$
\lim _{L \rightarrow \infty} S(L)=I_{f}+K_{f},
$$

where

$$
I_{f}=\int_{-\infty}^{\infty} \frac{d \theta}{4 \pi}\left(f(\theta)-G \frac{\cosh \theta}{\sinh ^{2} \theta}\right) \quad \text { and } \quad K_{f}=\frac{G}{4} \varphi(0) .
$$

The proof can be extracted from the appendix B of [37]. In addition, we also present a different derivation in appendix A. In the present case Theorem 1 can be applied with the substitutions

$$
f(\theta)=\cosh \theta K_{\alpha}(-\theta) K_{\beta}(\theta) e^{-2 m R \cosh \theta}, \quad G=\frac{g_{\alpha}^{2} g_{\beta}^{2}}{4} e^{-2 m R} .
$$

Here we made use of the identity $K_{\alpha}^{*}(\theta)=K_{\alpha}(-\theta)$. It is easy to see that with this choice $f(\theta)$ is indeed symmetric and it is analytic apart from the prescribed double pole at $\theta=0$.

The results of Theorem 1 for the Casimir energy yields

$$
\begin{aligned}
E_{0}(R)= & -m \frac{g_{\alpha} g_{\beta}}{4} e^{-m R}-m \frac{g_{\alpha}^{2} g_{\beta}^{2}}{4} e^{-2 m R} \frac{\varphi(0)}{4} \\
& -\frac{1}{2} \int \frac{d \theta}{2 \pi} m \cosh \theta\left(K_{\alpha}(-\theta) K_{\beta}(\theta) e^{-2 m R \cosh \theta}-\frac{g_{\alpha}^{2} g_{\beta}^{2}}{4 \sinh ^{2} \theta} e^{-2 m R}\right)+\mathcal{O}\left(e^{-3 m R}\right) .
\end{aligned}
$$

In order to compare this result to the BTBA equations it is necessary to derive a large $R$ expansion of (2.26). First of all we regularise the integral along the lines of [19]:

$$
E(R)=-m \frac{g_{\alpha} g_{\beta}}{4} \sqrt{e^{-\varepsilon(0)}}-\frac{1}{2} \int \frac{d \theta}{2 \pi} m \cosh \theta \log \left(\frac{1+K_{\alpha}(-\theta) K_{\beta}(\theta) e^{-\varepsilon(\theta)}}{1+\frac{g_{\alpha}^{2} g_{\beta}^{2}}{4 \sinh ^{2} \theta} e^{-\varepsilon(0)}}\right) .
$$

Using the same trick for the integral in (2.27) it is possible to derive the leading correction to the pseudo-energy:

$$
\varepsilon(\theta)=2 m R \cosh \theta-\frac{g_{\alpha} g_{\beta}}{2} \varphi(\theta) e^{-m R \cosh \theta}+\mathcal{O}\left(e^{-2 m R}\right),
$$

and in particular

$$
\sqrt{e^{-\varepsilon(0)}}=e^{-m R}\left(1+\frac{g_{\alpha} g_{\beta}}{4} \varphi(0) e^{-m R}+\ldots\right) .
$$


The second term in (2.33) can be expanded as

$$
\begin{aligned}
& \log \left(1+\frac{K_{\alpha}(-\theta) K_{\beta}(\theta) e^{-\varepsilon(\theta)}-\frac{g_{\alpha}^{2} g_{\beta}^{2}}{4 \sinh ^{2} \theta} e^{-\varepsilon(0)}}{1+\frac{g_{\alpha}^{2} g_{\beta}^{2}}{4 \sinh ^{2} \theta} e^{-\varepsilon(0)}}\right)= \\
& \quad=\frac{K_{\alpha}(-\theta) K_{\beta}(\theta) e^{-\varepsilon(\theta)}-\frac{g_{\alpha}^{2} g_{\beta}^{2}}{4 \sinh ^{2} \theta} e^{-\varepsilon(0)}}{1+\frac{g_{\alpha}^{2} g_{\beta}^{2}}{4 \sinh ^{2} \theta} e^{-\varepsilon(0)}} \approx\left(K_{\alpha}(-\theta) K_{\beta}(\theta) e^{-\varepsilon(\theta)}-\frac{g_{\alpha}^{2} g_{\beta}^{2}}{4 \sinh ^{2} \theta} e^{-\varepsilon(0)}\right) .
\end{aligned}
$$

In the last step we used the fact that the extra term in the denominator only makes a difference for $\theta \sim e^{-m R}$, therefore it only contributes to the higher order terms. In the last expression we may finally substitute the zeroth order approximation $\varepsilon(\theta) \approx 2 m R \cosh \theta$. Putting everything together we arrive at

$$
\begin{aligned}
E_{0}(R)= & -m \frac{g_{\alpha} g_{\beta}}{4} e^{-m R}-m \frac{g_{\alpha}^{2} g_{\beta}^{2}}{16} \varphi(0) e^{-2 m R} \\
& -\frac{1}{2} \int \frac{d \theta}{2 \pi} m \cosh \theta\left(K_{\alpha}(-\theta) K_{\beta}(\theta) e^{-2 m R \cosh (\theta)}-\frac{g_{\alpha}^{2} g_{\beta}^{2}}{4 \sinh ^{2} \theta} e^{-2 m R}\right)+\mathcal{O}\left(e^{-3 m R}\right) .
\end{aligned}
$$

This is in complete accordance with (2.32) and can be regarded as a strong confirmation of the consistency of our calculations. Apart from checking the identity $\bar{g}_{j}=g_{j} / 2$ and also the validity of the BTBA equations, we also confirmed the normalisation factor $N_{2}(\theta, L)$. In particular, a different $\mathcal{O}(1 / L)$ term in $N_{2}(\theta, L)$ would yield a different pre-factor for the term $\varphi(0) e^{-2 m R}$.

Finally we note that our finite volume regularisation scheme is not sensitive to the sign of $g_{\alpha} g_{\beta}$, in particular one arrives at the same result (2.37) also for $g_{\alpha} g_{\beta}<0$. On the other hand, in this regime one has to use the modified BTBA equations given by eq. (2.7) of [20]. We leave it as an exercise to show that a careful large $R$ expansion of this modified BTBA indeed reproduces (2.37).

\subsection{Connection between boundary states and form factors}

As a remark to this section we would like to point out an interesting similarity between the elementary finite volume form factors $\langle 0|O| \Psi\rangle_{L}$ and the amplitudes $g_{j}^{\Psi}(L)$ defined by

$$
\left\langle B_{j}^{L} \mid \Psi\right\rangle_{L}=e^{-f_{j} L} g_{j}^{\Psi}(L), \quad j=\alpha, \beta,
$$

where $f_{j}$ is the contribution of a single boundary to the ground state energy on a strip. Both quantities measure the overlap of a normalised eigenstate $|\Psi\rangle_{L}$ with a non-normalisable extended state, namely the boundary state $\left|B_{j}^{L}\right\rangle$ and the state $O|0\rangle_{L}$ created by acting with a local operator on the vacuum. To demonstrate the analogy we compare the main results in the case of the specific two-particle state $|\Psi\rangle_{L}=|\{I,-I\}\rangle_{L}$. We have seen that

$$
g_{j}^{\Psi}(L)=K_{j}(\theta) N_{2}(\theta, L) g_{j}^{0}(L)+\mathcal{O}\left(e^{-\mu L}\right) .
$$


The vacuum amplitude $g_{j}^{0}(L)$ only include exponentially small corrections (see below), therefore the excited state amplitude can be written as

$$
g_{j}^{\Psi}(L)=K_{j}(\theta) N_{2}(\theta, L)+\mathcal{O}\left(e^{-\mu L}\right) .
$$

On the other hand, the finite volume form factor was shown in [15] to be given by

$$
\langle 0|O| \Psi\rangle_{L}=\frac{F_{2}^{O}(\theta,-\theta)}{\sqrt{\rho_{2}(\theta,-\theta)}}+\mathcal{O}\left(e^{-\mu L}\right) .
$$

We observe that apart from the exponential corrections both objects are determined by the corresponding infinite volume quantity and a normalisation factor which only depends on the finite volume density of states. In the following we make some comments about the possible structure of the exponential corrections.

First of all it is instructive to recall the known results in the case of $|\Psi\rangle_{L}=|0\rangle_{L}$, which corresponds to the exact non-perturbative $g$-function and to the finite volume vacuum expectation value $\langle O\rangle_{L}$, respectively. In the case of the non-perturbative $g$-function the final result was expressed in [27] as

$$
\begin{aligned}
2 \log g_{j}^{0}(L)= & \frac{1}{2} \int \frac{d \theta}{2 \pi}\left(\Phi_{j}(\theta)-\delta(\theta)-2 \varphi(2 \theta)\right) \log \left(1+e^{-\varepsilon(\theta)}\right) \\
& +\sum_{i=1}^{\infty} \frac{1}{n} \int \frac{d \theta_{1}}{2 \pi} \ldots \int \frac{d \theta_{n}}{2 \pi}\left(\prod_{i=1}^{n} \frac{1}{1+e^{\varepsilon\left(\theta_{i}\right)}}\right) \varphi\left(\theta_{1}+\theta_{2}\right) \varphi\left(\theta_{2}-\theta_{3}\right) \ldots \varphi\left(\theta_{n}-\theta_{1}\right),
\end{aligned}
$$

where $\Phi_{j}(\theta)=-i \frac{d}{d \theta} \log \left(R_{j}(\theta)\right)$ and $\varepsilon(\theta)$ is the solution of the periodic-boundary-conditions TBA equation

$$
\varepsilon(\theta)=m L \cosh \theta-\int \frac{d \theta}{2 \pi} \varphi\left(\theta-\theta^{\prime}\right) \log \left(1+e^{-\varepsilon\left(\theta^{\prime}\right)}\right) .
$$

In the case of the vacuum expectation value the relevant exact result is the LeClairMussardo series [7]

$$
\langle\mathcal{O}\rangle_{L}^{\text {periodic }}=\sum_{n=0}^{\infty} \frac{1}{n !} \int \frac{d \theta_{1}}{2 \pi} \ldots \int \frac{d \theta_{n}}{2 \pi}\left(\prod_{i=1}^{n} \frac{1}{1+e^{\varepsilon\left(\theta_{i}\right)}}\right) F_{2 n, c}^{\mathcal{O}}\left(\theta_{1}, \ldots, \theta_{n}\right),
$$

where $\varepsilon(\theta)$ is the same pseudo-energy function as above. There is a striking structural similarity between the two series, most importantly the weight functions appearing in the integrals are exactly the same.

Based on these similarities we conjecture that the structure of exponential corrections to the excited state quantities $\langle 0|O| \Psi\rangle_{L}$ and $g_{j}^{\Psi}(L)$ will be similar to each other as well. It was remarked in [28] that the exact series for the amplitudes $g_{j}^{\Psi}(L)$ probably involves the solution of the corresponding excited state TBA. The appearance of the excited state TBA was also observed in preliminary studies of exponential corrections to form factors [38]. The exact result for $g_{j}^{\Psi}(L)$ will probably involve an integral series similar to (2.38) normalised by a "dressed" form of $N_{2}(\theta, L)$. 


\section{Evaluation of the vacuum expectation value}

In this section we develop a large $R$ expansion of the vacuum expectation value (v.e.v.) $\langle O\rangle_{R}^{\alpha, \beta}$. We use the finite volume regularisation scheme described in the previous section and express the expectation value as

$$
\langle O\rangle_{R}^{L}=\frac{\left\langle B_{\alpha}^{L}\left|e^{-H_{L} x} O e^{-H_{L}(R-x)}\right| B_{\beta}^{L}\right\rangle}{\left\langle B_{\alpha}^{L}\left|e^{-H_{L} R}\right| B_{\beta}^{L}\right\rangle} .
$$

We will calculate explicitly the v.e.v. with the boundary states truncated to contributions with up to four particles. In order to simplify notations we define

$$
\left|B_{j}^{L}\right\rangle=\sum_{n=0}^{4}\left|B_{j}^{L}\right\rangle^{(n)}, \quad j=\alpha, \beta,
$$

where

$$
\begin{aligned}
\left|B_{j}^{L}\right\rangle^{(0)} & =|0\rangle_{L}, \\
\left|B_{j}^{L}\right\rangle^{(1)} & =\frac{g_{j}}{2} N_{1}(L)|\{0\}\rangle_{L}, \\
\left|B_{j}^{L}\right\rangle^{(2)} & =\sum_{I} K_{j}(\theta) N_{2}(\theta, L)|\{I,-I\}\rangle_{L}, \\
\left|B_{j}^{L}\right\rangle^{(3)} & =\sum_{I} \frac{g_{j}}{2} K_{j}(\theta) N_{3}(\theta, L)|\{I,-I, 0\}\rangle_{L}, \\
\left|B_{j}^{L}\right\rangle^{(4)} & =\frac{1}{2} \sum_{\substack{I J \\
I \neq J}} K_{j}\left(\theta_{1}\right) K_{j}\left(\theta_{2}\right) N_{4}\left(\theta_{1}, \theta_{2}, L\right)|\{I,-I, J,-J\}\rangle_{L} .
\end{aligned}
$$

We also define

$$
C_{n m}={ }^{(n)}\left\langle B_{\alpha}^{L}\left|e^{-H_{L} x} O(x) e^{-H_{L}(R-x)}\right| B_{\beta}^{L}\right\rangle^{(m)} .
$$

In the following we perform a double expansion of (3.1) with expansion parameters $e^{-m x}$ and $e^{-m(R-x)}$. In order to keep track of the various terms let us introduce two auxiliary variables $u$ and $v$. They are use to count orders of $e^{-m x}$ and $e^{-m(R-x)}$ and at the end of the calculations both are set to 1 . Then the v.e.v. takes the form

$$
\langle O\rangle_{R}^{L}=\frac{1}{Z} \sum u^{n} v^{m} C_{n m}=\sum u^{n} v^{m} \tilde{D}_{n m} .
$$

We define

$$
Z=\sum_{n}(u v)^{n} Z_{n}
$$

where the first terms are given by

$$
Z_{0}=1, \quad Z_{1}=\frac{g_{\alpha} g_{\beta}}{4} m L e^{-m R},
$$

and

$$
Z_{2}=\sum_{I} e^{-2 m \cosh \theta R} K_{\alpha}^{*}(\theta) K_{\beta}(\theta) N_{2}(\theta, L)^{2}
$$


The inverse of the partition function is expanded as

$$
Z^{-1}=\sum_{n}(u v)^{n} \bar{Z}_{n}
$$

where the first few terms read

$$
\bar{Z}_{0}=1, \quad \bar{Z}_{1}=-Z_{1}, \quad \bar{Z}_{2}=Z_{1}^{2}-Z_{2} .
$$

Putting this together we obtain

$$
\tilde{D}_{n m}=\sum_{l} C_{n-l, m-l} \bar{Z}_{l}
$$

where the first few non-trivial terms are given by

$$
\begin{array}{ll}
\tilde{D}_{1 m}=C_{1 m}-Z_{1} C_{0, m-1}, & m=1,2, \ldots, \\
\tilde{D}_{2 m}=C_{2 m}-Z_{1} C_{1, m-1}+\left(Z_{1}^{2}-Z_{2}\right) C_{0, m-2}, & m=2,3, \ldots .
\end{array}
$$

We expect that the quantities $\tilde{D}_{n m}$ have a regular behaviour as $L \rightarrow \infty$ and for the actual limit we define

$$
D_{n m}=\lim _{L \rightarrow \infty} \tilde{D}_{n m} .
$$

The vacuum expectation value is then expressed as

$$
\langle O\rangle_{R}^{\alpha, \beta}=\sum_{n, m} D_{n m}
$$

Note that the individual terms contributing to $\tilde{D}_{n m}$ may contain divergent pieces which scale with positive powers of $L$. The most singular terms in the $n$ particle sector carry a factor of $\left(m L e^{-m R}\right)^{n}$, therefore the expansion is valid in the regime

$$
1 \ll m L \ll e^{m R} .
$$

The $L \rightarrow \infty$ limit of the complete series (3.3) is to be understood as an analytic continuation.

The evaluation of the individual $C_{n m}$ is built on the knowledge of the finite volume form factors. In the general case they are given by [15]

$$
\left\langle\left\{I_{1}, \ldots, I_{n}\right\}|O|\left\{J_{1}, \ldots, J_{m}\right\}\right\rangle_{L}=\frac{F_{n+m}^{O}\left(\theta_{1}+i \pi, \ldots, \theta_{n}+i \pi, \theta_{1}^{\prime}, \ldots, \theta_{m}^{\prime}\right)}{\sqrt{\rho_{n}\left(\theta_{1}, \ldots, \theta_{n}\right) \rho_{m}\left(\theta_{1}^{\prime}, \ldots, \theta_{m}^{\prime}\right)}}+\mathcal{O}\left(e^{-\mu L}\right),
$$

where it is understood that the rapidities $\left\{\theta_{1}, \ldots, \theta_{n}\right\}$ and $\left\{\theta_{1}^{\prime}, \ldots, \theta_{m}^{\prime}\right\}$ are solutions to the corresponding Bethe-Yang equations. Formula (3.8) is valid whenever there are no coinciding rapidities in the two scattering states. In [15] it was shown that the only two situations when coinciding rapidities occur are the case of diagonal form factors and matrix elements between parity-symmetric states containing zero-momentum particles. The general rule for evaluating disconnected contributions can be found in [11]; in the present work we will cite the necessary results whenever they are needed. 
Before turning to the actual calculations we have to address a very important question concerning the phase of the form factors. Note that equation (3.8) is to be understood up to a phase factor; in particular the order of the rapidities is not determined by first principles. In fact, an additional phase factor corresponds to a redefinition of the basis vectors, and this phase drops out from the calculation of some physical quantities, for example correlation functions in infinite volume. However, phase factors are utterly relevant in the present case because they do affect the final result for the v.e.v. A guideline can be established from the known results in infinite volume, where the correct phase is fixed by consistency arguments. First of all, the two-particle contributions to the boundary state are written as

$$
K_{j}(\theta) A(-\theta) A(\theta)
$$

This expression is symmetric in $\theta$ as required by consistency. When it comes to the evaluation of the v.e.v. in the presence of a single boundary, the above definition yields the expressions

$$
\ldots K_{\beta}(\theta) F_{n}^{O}(-\theta, \theta, \ldots) \text { and } \ldots K_{\alpha}^{*}(\theta) F_{n}^{O}(\theta+i \pi,-\theta+i \pi, \ldots)
$$

where the dots stand for possible additional rapidities and amplitudes. Observe that both expressions above are symmetric in $\theta$, therefore we apply the following rule: whenever there appear the amplitudes $K_{\beta}\left(\theta_{i}\right)$ and $K_{\alpha}^{*}\left(\theta_{i}\right)$ with some $\theta_{i}$, the explicit form and order of the rapidities substituted into the relevant form factor is given by $\left(-\theta_{i}, \theta_{i}\right)$ and $\left(\theta_{i}+i \pi,-\theta_{i}+i \pi\right)$, respectively. The exchange of two pairs of rapidities does not make a difference, therefore the phase of the form factors is completely fixed by the above rule.

The complex conjugation of the amplitude $K_{\alpha}$ can be avoided by means of the identity

$$
K_{\alpha}^{*}\left(\theta_{i}\right)=K_{\alpha}\left(-\theta_{i}\right)
$$

An additional change of variables $\theta_{i} \rightarrow-\theta_{i}$ then amounts to the convention

$$
\ldots K_{\alpha}(\theta) F_{n}^{O}(-\theta+i \pi,+\theta+i \pi, \ldots)
$$

Note also that the presence of zero-momentum particles does not produce any additional ambiguities, because it is only the expressions

$$
K_{j}(\theta) F_{n}^{O}(-\theta, \theta, 0, \ldots)=K_{j}(\theta) F_{n}^{O}(0,-\theta, \theta, \ldots)
$$

which are symmetric in $\theta$, i.e. the zero-momentum particle cannot be placed between the particles $A(\theta)$ and $A(-\theta)$.

In the following we evaluate all $D_{n m}$ with $n+m \leq 4$. We put forward that all our results are collected in appendix $\mathrm{C}$ together with a pictorial representation of the individual terms. 
The contributions $D_{n 0}$ and $D_{0 m}$ with $n, m=1 \ldots 4$ only depend on one of the boundaries and they are the same as obtained in [13]:

$$
\begin{aligned}
D_{10} & =\frac{g_{\alpha}}{2} F_{1}^{O} e^{-m x} \\
D_{20} & =\frac{1}{2} \int \frac{d \theta}{2 \pi} K_{\alpha}(\theta) F_{2}^{O}(-\theta, \theta) e^{-2 m \cosh \theta x}, \\
D_{30} & =\frac{1}{2} \int \frac{d \theta}{2 \pi} K_{\alpha}(\theta) \frac{g_{\alpha}}{2} F_{3}^{O}(-\theta, \theta, 0) e^{-m(2 \cosh \theta+1) x}, \\
D_{40} & =\frac{1}{8} \int \frac{d \theta_{1}}{2 \pi} \frac{d \theta_{2}}{2 \pi} K_{\alpha}\left(\theta_{1}\right) K_{\alpha}\left(\theta_{2}\right) F_{4}^{O}\left(-\theta_{1}, \theta_{1},-\theta_{2}, \theta_{2}\right) e^{-2 m\left(\cosh \theta_{1}+\cosh \theta_{2}\right) x} \\
D_{01} & =\frac{g_{\beta}}{2} F_{1}^{O} e^{-m(R-x)} \\
D_{02} & =\frac{1}{2} \int \frac{d \theta}{2 \pi} K_{\beta}(\theta) F_{2}^{O}(-\theta, \theta) e^{-2 m \cosh \theta(R-x)}, \\
D_{03} & =\frac{1}{2} \int \frac{d \theta}{2 \pi} K_{\beta}(\theta) \frac{g_{\beta}}{2} F_{3}^{O}(-\theta, \theta, 0) e^{-m(2 \cosh \theta+1)(R-x)}, \\
D_{04} & =\frac{1}{8} \int \frac{d \theta_{1}}{2 \pi} \frac{d \theta_{2}}{2 \pi} K_{\beta}\left(\theta_{1}\right) K_{\beta}\left(\theta_{2}\right) F_{4}^{O}\left(-\theta_{1}, \theta_{1},-\theta_{2}, \theta_{2}\right) e^{-2 m\left(\cosh \theta_{1}+\cosh \theta_{2}\right)(R-x)} .
\end{aligned}
$$

Note that these integrals remain well-defined even if there are poles in the amplitudes $K_{j}(\theta)$, because the form factors possess the appropriate number of zeros at $\theta_{i}=0$ as a consequence of the exchange axiom (2.9).

The first contribution to contain a divergent piece is $D_{11}$ which is given by

$$
D_{11}=\lim _{L \rightarrow \infty}\left(C_{11}-Z_{1} C_{00}\right)
$$

where

$$
C_{11}=\frac{g_{\alpha} g_{\beta}}{4} m L\langle\{0\}|O|\{0\}\rangle_{L} e^{-m R} .
$$

The diagonal one-particle form factor for a generic $I$ is given by [11]

$$
\langle\{I\}|O|\{I\}\rangle_{L}=\frac{F_{2}^{O}(i \pi+\theta, \theta)}{\rho_{1}(\theta)}+\langle O\rangle .
$$

The two-particle form factor appearing in the expression above is free of divergences and by Lorentz-invariance it does not depend on $\theta$. In the following it will be denoted by $F_{2, s}^{O}$. Specifying (3.11) to $I=0$ one finds

$$
D_{11}=\lim _{L \rightarrow \infty}\left(C_{11}-Z_{1} C_{00}\right)=\frac{g_{\alpha} g_{\beta}}{4} F_{2, s}^{O} e^{-m R} .
$$

\subsection{Evaluation of $D_{21}$ and $D_{12}$}

We first consider

$$
D_{21}=\lim _{L \rightarrow \infty}\left(C_{21}-Z_{1} C_{10}\right)
$$

with

$$
C_{21}=\frac{g_{\beta}}{2} \sum_{I} \frac{F_{3}^{O}(-\theta+i \pi, \theta+i \pi, 0)}{\bar{\rho}_{1}(\theta)} e^{-E_{I} x-m(R-x)} K_{\alpha}(\theta),
$$


where it is understood that the rapidities $\theta$ are solutions of the corresponding Bethe-Yang equations

$$
m L \sinh \theta+\delta(\theta)+\delta(2 \theta)=2 \pi I,
$$

and the constrained density is

$$
\bar{\rho}_{1}(\theta)=m L \cosh \theta+2 \varphi(2 \theta) .
$$

Note also that we have already made use of the relations (2.22). Naively one would take the $L \rightarrow \infty$ limit simply by replacing the summation with and integration leading to

$$
\frac{1}{2} \frac{g_{\beta}}{2} \int \frac{d \theta}{2 \pi} F_{3}^{O}(-\theta+i \pi, \theta+i \pi, 0) e^{-2 m \cosh \theta x-m(R-x)} K_{\alpha}(\theta) .
$$

However, the expression above is ill-defined at $\theta=0$ due to the poles of $F_{3}$ and $K_{\alpha}$. Not surprisingly, this divergence gets cancelled by $Z_{1} C_{10}$ as we will show shortly.

It follows from the form factor axioms (2.9) and (2.11) that near $\theta_{1,2}=0$

$$
F_{3}^{O}\left(\theta_{1}+i \pi, \theta_{2}+i \pi, 0\right) \approx 2 i F_{1}^{O}\left(\frac{1}{\theta_{2}}-\frac{1}{\theta_{1}}\right)
$$

and therefore

$$
F_{3}^{O}(-\theta+i \pi, \theta+i \pi, 0) \approx 4 i F_{1}^{O} \frac{1}{\theta}+\ldots
$$

The singular behaviour of the sum (3.14) is determined by the states near $\theta=0$. We use the approximation of section 2.2 to determine the leading divergence as

$$
4 \frac{F_{1}^{O}}{m L} \frac{\left(g_{\alpha}\right)^{2} g_{\beta}}{4}\left(\frac{m L}{2 \pi}\right)^{2} e^{-m(x+R)} \sum_{I}\left(\frac{1}{I}\right)^{2}=4 \frac{F_{1}^{O}}{m L} \frac{\left(g_{\alpha}\right)^{2} g_{\beta}}{4}\left(\frac{m L}{2 \pi}\right)^{2} e^{-m(x+R)} \frac{\pi^{2}}{2} .
$$

This coincides with $Z_{1} C_{10}$ therefore (3.13) has a finite $L \rightarrow \infty$ limit indeed.

Similarly to the calculations of section 2.2 we use Theorem 1 to obtain the finite-left over piece in (3.13). We apply the substitutions

$$
f(\theta)=F_{3}^{O}(-\theta+i \pi, \theta+i \pi, 0) e^{-2 m \cosh \theta x-m(R-x)} K_{\alpha}(\theta), \quad G=2\left(g_{\alpha}\right)^{2} F_{1}^{O} e^{-m(x+R)} .
$$

It is easy to see that $f(\theta)$ is indeed a symmetric function, therefore its only singularity near the real axis is a double pole at $\theta=0$. The net result is then expressed as

$$
\begin{aligned}
D_{21}= & \frac{g_{\beta}}{4} \int \frac{d \theta}{2 \pi}\left(F_{3}^{O}(-\theta+i \pi, \theta+i \pi, 0) K_{\alpha}(\theta) e^{-2 m \cosh \theta x-m(R-x)}-\frac{2\left(g_{\alpha}\right)^{2} F_{1}^{O} \cosh \theta}{\sinh ^{2} \theta} e^{-m(R+x)}\right) \\
& +e^{-m(x+R)} g_{\beta}\left(g_{\alpha}\right)^{2} F_{1}^{O} \frac{\varphi(0)}{4} .
\end{aligned}
$$

A similar calculation with the roles of the two boundaries exchanged yields

$$
\begin{aligned}
D_{12}= & \frac{g_{\alpha}}{4} \int \frac{d \theta}{2 \pi}\left(F_{3}^{O}(i \pi,-\theta, \theta) K_{\beta}(\theta) e^{-2 m \cosh \theta(R-x)-m x}-\frac{2\left(g_{\beta}\right)^{2} F_{1}^{O} \cosh \theta}{\sinh ^{2} \theta} e^{-m(2 R-x)}\right) \\
& +e^{-m(2 R-x)} g_{\alpha}\left(g_{\beta}\right)^{2} F_{1}^{O} \frac{\varphi(0)}{4} .
\end{aligned}
$$




\subsection{Evaluation of $D_{13}$ and $D_{31}$}

We first consider $D_{31}=\lim _{L \rightarrow \infty}\left(C_{31}-Z_{1} C_{20}\right)$ where

$$
\begin{aligned}
C_{31}-Z_{1} C_{20}=\sum_{I} N_{3}(\theta) \sqrt{m L} \frac{g_{\alpha} g_{\beta}}{4} K_{\alpha}^{*}(\theta)\langle\{I,-I, 0\}|\mathcal{O}|\{0\}\rangle_{L} e^{-m R-2 m \cosh \theta x_{-}} \\
\quad-\frac{g_{\alpha} g_{\beta}}{4} m L e^{-m R} \sum_{J} N_{2}(\theta)\langle\{J,-J\}|O| 0\rangle_{L} e^{-E_{I} x} K_{\alpha}^{*}(\theta) .
\end{aligned}
$$

The three-particle normalisation is given by

$$
N_{3}(\theta)=\frac{\sqrt{\rho_{3}(\theta,-\theta, 0)}}{\bar{\rho}_{3}(\theta)} .
$$

The four-particle matrix element above includes a disconnected term due to the zeromomentum particles which is given by [11]

$$
\langle\{I,-I, 0\}|\mathcal{O}|\{0\}\rangle_{L}=\frac{F_{4}^{O}(\theta+i \pi,-\theta+i \pi, i \pi, 0)+m L F_{2}^{O}(\theta,-\theta)}{\sqrt{\rho_{3}(\theta,-\theta, 0) m L}},
$$

while the connected part of $C_{31}$ can be transformed into the integral

$$
\frac{g_{\alpha} g_{\beta}}{8} e^{-m R} \int \frac{d \theta}{2 \pi} K_{\alpha}(\theta) F_{4}^{O}(-\theta+i \pi, \theta+i \pi, i \pi, 0) e^{-2 m \cosh \theta x} .
$$

It follows from the form factor axioms that $F_{4}^{O}(-\theta+i \pi, \theta+i \pi, i \pi, 0)$ is well-defined [11] and has a regular behaviour as a function of $\theta$. Therefore we can apply the exchange axiom (2.9) to show that

$$
\lim _{\theta \rightarrow 0} F_{4}^{O}(-\theta+i \pi, \theta+i \pi, i \pi, 0)=0
$$

therefore the integral (3.21) is regular even at $\theta=0$.

The divergent pieces of $D_{31}$ are given by

$$
\frac{g_{\alpha} g_{\beta}}{4} e^{-m R} m L\left(\sum_{I} \frac{F_{2}^{O}(-\theta, \theta)}{\bar{\rho}_{3}(\theta)} K_{\alpha}(\theta) e^{-2 m \cosh \theta x}-\sum_{J} \frac{F_{2}^{O}(-\theta, \theta)}{\bar{\rho}_{1}(\theta)} K_{\alpha}(\theta) e^{-2 m \cosh \theta x}\right) .
$$

Note the summations over $I$ and $J$ run over the solutions of the different quantisation conditions (2.18) and (2.25), respectively. However, the density factors are the corresponding ones in both sum, therefore it is allowed to replace the summation with an integration in both terms. Doing this we make errors of order $e^{-\mu L}$ because both integrands are regular, therefore the above expression vanishes in the $L \rightarrow \infty$ limit and the net result is

$$
D_{31}=\frac{g_{\alpha} g_{\beta}}{8} e^{-m R} \int \frac{d \theta}{2 \pi} K_{\alpha}(\theta) F_{4}^{O}(-\theta+i \pi, \theta+i \pi, i \pi, 0) e^{-2 m \cosh \theta x},
$$

and similarly,

$$
D_{13}=\frac{g_{\alpha} g_{\beta}}{8} e^{-m R} \int \frac{d \theta}{2 \pi} K_{\beta}(\theta) F_{4}^{O}(-\theta+i \pi, \theta+i \pi, i \pi, 0) e^{-2 m \cosh \theta(R-x)} .
$$




\subsection{Evaluation of $D_{22}$}

Here we consider

$$
D_{22}=\lim _{L \rightarrow \infty}\left(C_{22}-Z_{2}\langle O\rangle-Z_{1} C_{11}+\left(Z_{1}\right)^{2}\langle O\rangle\right) .
$$

Evaluating $C_{22}$ one has to treat the diagonal and off-diagonal matrix elements separately:

$$
\begin{aligned}
C_{22}=\sum_{I \neq J} N_{2}\left(\theta_{1}\right) N_{2}\left(\theta_{2}\right) & \{I,-I\}|O|\{J,-J\}\rangle_{L} e^{-E_{I} x-E_{J}(R-x)} K_{\alpha}\left(-\theta_{1}\right) K_{\beta}\left(\theta_{2}\right)+ \\
& +\sum_{I} N_{2}(\theta)^{2}\langle\{I,-I\}|O|\{I,-I\}\rangle_{L} K_{\alpha}(-\theta) K_{\beta}(\theta) e^{-E_{I} R} .
\end{aligned}
$$

Here it is understood that the rapidities $\theta_{1}$ and $\theta_{2}$ solve the quantisation condition (2.18) with momentum quantum numbers $I$ and $J$, respectively (including the diagonal case with $\theta_{1}=\theta_{2}$ and $\left.I=J\right)$. In the off-diagonal case one has

$$
N_{2}\left(\theta_{1}\right) N_{2}\left(\theta_{2}\right)\langle\{I,-I\}|O|\{J,-J\}\rangle_{L}=\frac{F_{4}^{O}\left(\theta_{1}+i \pi,-\theta_{1}+i \pi,-\theta_{2}, \theta_{2}\right)}{\bar{\rho}_{1}\left(\theta_{1}\right) \bar{\rho}_{1}\left(\theta_{2}\right)} .
$$

It would be desirable to convert the sum over these matrix elements into an integral. However, one has to be careful because of the ambiguity of the form factors near $\theta_{1}=\theta_{2}$ and the possible poles of the integrand at $\theta_{1}=\theta_{2}=0$. As a first step we write

$$
\begin{aligned}
& \sum_{I \neq J} \frac{F_{4}^{O}\left(\theta_{1}+i \pi,-\theta_{1}+i \pi,-\theta_{2}, \theta_{2}\right)}{\bar{\rho}_{1}\left(\theta_{1}\right) \bar{\rho}_{1}\left(\theta_{2}\right)} K_{\alpha}\left(-\theta_{1}\right) K_{\beta}\left(\theta_{2}\right) e^{-2 m \cosh \theta_{1} x-2 m \cosh \theta_{2}(R-x)}= \\
& =\frac{1}{4} \int \frac{d \theta_{1}}{2 \pi} \int \frac{d \theta_{2}}{2 \pi} K_{\alpha}\left(-\theta_{1}\right) K_{\beta}\left(\theta_{2}\right) F_{4}^{O}\left(\theta_{1}+i \pi,-\theta_{1}+i \pi,-\theta_{2}, \theta_{2}\right) e^{-2 m \cosh \theta_{1} x-2 m \cosh \theta_{2}(R-x)} \\
& \quad-\sum_{I} \frac{\bar{F}_{4}^{O}(\theta+i \pi,-\theta+i \pi,-\theta, \theta)}{\bar{\rho}_{1}(\theta)^{2}} K_{\alpha}(-\theta) K_{\beta}(\theta) e^{-E_{I} R}+\mathcal{O}\left(e^{-\mu L}\right),
\end{aligned}
$$

where $\bar{F}_{4}^{O}(\theta+i \pi,-\theta+i \pi,-\theta, \theta)$ is the continuation of the function $F_{4}^{O}\left(\theta_{1}+i \pi,-\theta_{1}+\right.$ $\left.i \pi,-\theta_{2}, \theta_{2}\right)$ to $\theta_{1}=\theta_{2}=\theta$. At first sight there seems to be a double kinematic pole, however this is not quite true. In [11] it was shown that diagonal form factors may be assigned a finite value, although the diagonal limit will depend on the particular evaluation method used. In the following we restate the results of [11] which are needed to evaluate (3.27).

Consider the quantity

$$
F_{2 n}^{O}\left(\theta_{1}+\varepsilon_{1}+i \pi, \ldots, \theta_{n}+\varepsilon_{n}+i \pi, \theta_{n}, \ldots, \theta_{1}\right),
$$

where the singularities have been shifted off by the infinitesimal quantities $\varepsilon_{i}$. It was proven in [11] that there exists a finite limit when all $\varepsilon_{i}$ go to zero simultaneously with their ratios fixed. Moreover, there are two special evaluation schemes which respect the physical requirement that diagonal form factors should not depend on the order of the rapidities. First of all, one can consider the symmetric limit with $\varepsilon_{1}=\varepsilon_{2}=\cdots=\varepsilon$ :

$$
F_{2 n, s}^{O}\left(\theta_{1}, \ldots, \theta_{n}\right) \equiv \lim _{\varepsilon \rightarrow 0} F_{2 n}^{O}\left(\theta_{1}+\varepsilon+i \pi, \ldots, \theta_{n}+\varepsilon+i \pi, \theta_{n}, \ldots, \theta_{1}\right) .
$$


On the other hand, one can also consider the connected part of the diagonal form factor which is defined to be the contribution to (3.28) which does not contain any singular factors of the form $\varepsilon_{i} / \varepsilon_{j}$ and products thereof:

$$
F_{2 n, c}^{O}\left(\theta_{1}, \ldots, \theta_{n}\right) \equiv \text { (finite part of) } F_{2 n}^{O}\left(\theta_{1}+\varepsilon_{1}+i \pi, \ldots, \theta_{n}+\varepsilon_{n}+i \pi, \theta_{n}, \ldots, \theta_{1}\right) .
$$

The general structure of the singularities in (3.28) together with the relation between the symmetric and connected evaluation schemes was worked out in [11]. Here we only need the formula relevant to the two-particle states, which states that given two infinitesimal numbers $\varepsilon_{1}$ and $\varepsilon_{2}$ one has

$$
F_{4}^{O}\left(\theta_{1}+\varepsilon_{1}+i \pi,-\theta_{2}+\varepsilon_{2}+i \pi,-\theta_{2}, \theta_{1}\right)=F_{4, c}^{O}\left(\theta_{1}, \theta_{2}\right)+\left(\frac{\varepsilon_{1}}{\varepsilon_{2}}+\frac{\varepsilon_{2}}{\varepsilon_{1}}\right) \varphi\left(\theta_{1}-\theta_{2}\right) F_{2, s}^{O} .
$$

In particular, the symmetric evaluation with $\varepsilon_{1}=\varepsilon_{2}$ is given by

$$
F_{4, s}^{O}\left(\theta_{1}, \theta_{2}\right)=F_{4, c}^{O}\left(\theta_{1}, \theta_{2}\right)+2 \varphi\left(\theta_{1}-\theta_{2}\right) F_{2, s}^{O} .
$$

In the present case we need to evaluate $F_{4}^{O}\left(\theta_{1}+i \pi,-\theta_{1}+i \pi,-\theta_{2}, \theta_{2}\right)$ near $\theta_{1}=\theta_{2}=\theta$ which corresponds to an "antisymmetric evaluation" with $\varepsilon_{2}=-\varepsilon_{1}$ :

$$
\begin{aligned}
\bar{F}_{4}^{O}(\theta+i \pi,-\theta+i \pi,-\theta, \theta) & \equiv \lim _{\varepsilon \rightarrow 0} F_{4}^{O}(\theta+\varepsilon+i \pi,-\theta-\varepsilon+i \pi,-\theta, \theta) \\
& =F_{4, c}^{O}(\theta,-\theta)-2 \varphi(2 \theta) F_{2, s}^{O} .
\end{aligned}
$$

Note that the integral in (3.27) is well-defined even if there are poles in the factors $K_{\alpha}(-\theta) K_{\beta}(\theta)$ because $F_{4}^{O}\left(\theta_{1}+i \pi,-\theta_{1}+i \pi,-\theta_{2}, \theta_{2}\right)$ possesses a double zero at $\theta_{1}=\theta_{2}=0$ (for a proof see appendix B).

In order to evaluate $C_{22}$ we also need the diagonal finite volume form factors. A generic diagonal four-particle matrix element reads [11]

$$
\left\langle\left\{I_{1}, I_{2}\right\}|O|\left\{I_{1}, I_{2}\right\}\right\rangle_{L}=\frac{F_{4, s}^{O}\left(\theta_{1}, \theta_{2}\right)+\left(\rho_{1}\left(\theta_{1}\right)+\rho_{1}\left(\theta_{2}\right)\right) F_{2, s}^{O}}{\rho_{2}\left(\theta_{1}, \theta_{2}\right)}+\langle O\rangle .
$$

Specifying the above formula to the present case one obtains

$$
\langle\{-I, I\}|O|\{-I, I\}\rangle_{L}=\frac{F_{4, s}^{O}(\theta,-\theta)+2 \rho_{1}(\theta) F_{2, s}^{O}}{\rho_{2}(\theta,-\theta)}+\langle O\rangle .
$$

Substituting the results of (3.27), (3.32) and (3.34) into (3.25) we obtain

$$
\begin{aligned}
& \left(C_{22}-Z_{2}\langle O\rangle-Z_{1} C_{11}+\left(Z_{1}\right)^{2}\langle O\rangle\right)= \\
& =\frac{1}{4} \int \frac{d \theta_{1}}{2 \pi} \int \frac{d \theta_{2}}{2 \pi} K_{\alpha}\left(-\theta_{1}\right) K_{\beta}\left(\theta_{2}\right) F_{4}^{O}\left(\theta_{1}+i \pi,-\theta_{1}+i \pi,-\theta_{2}, \theta_{2}\right) e^{-2 m \cosh \theta_{1} x-2 m \cosh \theta_{2}(R-x)} \\
& \quad+F_{2, s}^{O}\left(\sum_{I} \frac{2}{\bar{\rho}_{1}(\theta)} K_{\alpha}(-\theta) K_{\beta}(\theta) e^{-2 m \cosh \theta R}-\frac{\left(g_{\alpha} g_{\beta}\right)^{2}}{16} m L e^{-2 m R}\right)+\mathcal{O}\left(e^{-\mu L}\right),
\end{aligned}
$$

where we applied the normalisation given by $(2.22)$ and we also made use of the relation (3.31) and the identity

$$
\bar{\rho}_{1}(\theta)=\rho(\theta)+2 \varphi(2 \theta) .
$$


The summation in the third line of (3.35) still contains a divergent $\mathcal{O}(L)$ piece. However, it can be proven along the lines of the previous subsections, that this divergence gets exactly cancelled. One can use Theorem 1 with the substitutions

$$
f(\theta)=2 K_{\alpha}(-\theta) K_{\beta}(\theta) e^{-2 m \cosh \theta R}, \quad G=\frac{\left(g_{\alpha} g_{\beta}\right)^{2}}{2} e^{-2 m R}
$$

to obtain the finite left-over piece. The net result reads

$$
D_{22}=\lim _{L \rightarrow \infty}\left(C_{22}-Z_{2}\langle O\rangle-Z_{1} C_{11}+\left(Z_{1}\right)^{2}\langle O\rangle\right)=I_{22}+J_{22},
$$

where

$$
I_{22}=\frac{1}{4} \int \frac{d \theta_{1}}{2 \pi} \frac{d \theta_{2}}{2 \pi} K_{\alpha}\left(\theta_{1}\right) K_{\beta}\left(\theta_{2}\right) F_{4}^{O}\left(-\theta_{1}+i \pi, \theta_{1}+i \pi,-\theta_{2}, \theta_{2}\right) e^{-2 m \cosh \theta_{1} x-2 m \cosh \theta_{2}(R-x)}
$$

and

$$
J_{22}=F_{2, s}^{O} \int \frac{d \theta}{2 \pi}\left(K_{\alpha}(-\theta) K_{\beta}(\theta) e^{-2 m \cosh \theta R}-\frac{\left(g_{\alpha} g_{\beta}\right)^{2} \cosh \theta}{4 \sinh ^{2} \theta} e^{-2 m R}\right)+F_{2, s}^{O} \frac{\left(g_{\alpha} g_{\beta}\right)^{2}}{8} e^{-2 m R} \varphi(0) .
$$

With this we have finished the calculation of the terms in (3.3) with $n+m \leq 4$. The reader can find the various terms collected in appendix $\mathrm{C}$ together with a pictorial representation of them.

\subsection{Connections to other problems}

In this subsection we compare our results to those obtained in previous works. Also, we establish a connection to the problem of finite temperature correlation functions.

\subsubsection{Comparison with a proposal of LeClair et al.}

The first result in the literature to deal with expectation values on a strip appeared in [4]. In appendix $\mathrm{C}$ the authors conjecture the general result to be

$$
\langle O(x)\rangle_{R}=\sum_{n=0}^{\infty} \sum_{\varepsilon_{i}= \pm} \frac{1}{n !} I_{\varepsilon_{1}, \ldots, \varepsilon_{n}}^{(n)}
$$

where

$$
I_{\varepsilon_{1}, \ldots, \varepsilon_{n}}^{(n)}=\prod_{i=1}^{n}\left\{\int_{-\infty}^{\infty} \frac{d \theta_{i}}{4 \pi} g_{\varepsilon_{i}}\left(\theta_{i}\right)\right\} F_{2 n}^{O}\left(\theta_{1},-\theta_{1}, \ldots, \theta_{n},-\theta_{n}\right)_{\varepsilon_{1}, \ldots, \varepsilon_{n}} .
$$

The weight functions above are defined by

$$
g_{+}(\theta)=\frac{e^{-2 m x \cosh \theta} K_{\alpha}(-\theta)}{1+K_{\alpha}(-\theta) K_{\beta}(\theta) e^{-2 m R \cosh \theta}}, \quad g_{-}(\theta)=\frac{e^{-2 m(R-x) \cosh \theta} K_{\beta}(\theta)}{1+K_{\alpha}(-\theta) K_{\beta}(\theta) e^{-2 m R \cosh \theta}},
$$

and the form factors are given by

$$
F_{2 n}^{O}\left(\theta_{1},-\theta_{1}, \ldots, \theta_{n},-\theta_{n}\right)_{\varepsilon_{1}, \ldots, \varepsilon_{n}} \equiv F_{2 n}^{O}\left(\theta_{1}+i \bar{\sigma}_{1},-\theta_{1}+i \bar{\sigma}_{1}, \ldots, \theta_{n}+i \bar{\sigma}_{n},-\theta_{n}+i \bar{\sigma}_{n}\right)
$$

with $\bar{\sigma}_{i}=\pi\left(1+\varepsilon_{i}\right) / 2$. It is assumed that $g_{\alpha}=g_{\beta}=0$. 
It is easy to see that some of our results are reproduced by the above series. However, there is also a serious discrepancy: (3.39) does not include the diagonal contribution $J_{22}$ given by (3.38). This can be proven by putting $x=R / 2$ and considering the large $R$ limit of (3.39). In this case the $n$th term behaves as $\mathcal{O}\left(e^{-m n R}\right)$ while $J_{22}$ is of order $e^{-2 m R}$. Therefore $J_{22}$ can only appear in the terms $n \leq 2$. On the other hand these contributions yield

$$
\begin{array}{rlrl}
I_{+}^{(1)} & =D_{20}+\mathcal{O}\left(e^{-3 m R}\right), & I_{-}^{(1)} & =D_{02}+\mathcal{O}\left(e^{-3 m R}\right), \\
I_{++}^{(2)} & =D_{40}+\mathcal{O}\left(e^{-4 m R}\right), & I_{--}^{(2)} & =D_{04}+\mathcal{O}\left(e^{-4 m R}\right), \\
I_{+-}^{(2)} & =I_{-+}^{(2)}=I_{22}+\mathcal{O}\left(e^{-4 m R}\right) . &
\end{array}
$$

All the above terms are well-defined and finite, thus it follows that $J_{22}$ is not included in (3.39).

We will demonstrate in section 4.2 with the help of non-perturbative numerical results that the term $J_{22}$ must be included in the expansion, thus the series (3.39) can not be complete in the general case.

\subsubsection{Relation to quench problems}

In recent papers $[2,3]$ the integrable QFT approach was applied to quench problems. In particular, they considered the time evolution of expectation values of local operators after a sudden global quench which changes the Hamiltonian from $H_{0}$ to $H$, where $H$ (possibly also $H_{0}$ ) is considered to be integrable. The main assumption is that the initial state of the system (which is the ground state of $H_{0}$ ) can be expanded in the multi-particle basis of the integrable Hamiltonian $H$ as a boundary state. In the simplest case with only one particle type in the spectrum the corresponding expression is

$$
|B\rangle=\exp \left(\int \frac{d \theta}{4 \pi} K(\theta) A(-\theta) A(\theta)\right)|0\rangle
$$

where $K(\theta)$ is an arbitrary function satisfying $K(\theta)=S(2 \theta) K(-\theta)$. The time evolution of an expectation value is then given by

$$
\langle O(0, t)\rangle=\left\langle B\left|e^{i H t} O(0,0) e^{-i H t}\right| B\right\rangle .
$$

It is straightforward to establish a connection between the real-time evolution of the expectation value $\langle O(0, t)\rangle$ and the static (Euclidean) quantity $\langle O(x)\rangle_{R}^{\alpha \beta}$ considered in this work. In fact, the quench problem above corresponds to the choice

$$
K_{\alpha}(\theta)=K_{\beta}(\theta)=K(\theta),
$$

and the expectation value $\langle O(0, t)\rangle$ is obtained by the analytic continuation

$$
R=2 \tau_{0}, \quad x=\tau_{0}-i t,
$$

where $\tau_{0}$ is a constant which is analogous to the extrapolation length introduced in the field theory approach to boundary critical phenomena $[1,3] .^{5}$

\footnotetext{
${ }^{5}$ Keeping a finite (non-zero) $\tau_{0}$ is necessary to normalise the boundary states properly, otherwise the spectral series will not be convergent.
} 
The results of [2] can be reproduced by our expansion with the analytic continuation explained above. The main difference between [2] and the present work is that [2] concerns the sine-Gordon model which is a non-diagonal scattering theory. However, the authors calculate contributions only from low-lying breather states which possess diagonal scattering. Therefore our methods directly apply to the situation in [2]. Moreover, our expansion includes higher order terms which were not considered in [2].

In the following we also compare our results to those of [3], which considers the $t \rightarrow \infty$ limit of the expectation value $\langle O(0, t)\rangle$. For this quantity the authors obtain the series expansion

$$
\langle O\rangle=\sum_{n=0}^{\infty} \frac{1}{n !} \prod_{i}\left\{\int \frac{d \theta_{i}}{2 \pi} \frac{\left|G\left(\theta_{i}\right)\right|^{2}}{1+\left|G\left(\theta_{i}\right)\right|^{2}}\right\} F_{2 n, c}^{O}\left(\theta_{1}, \ldots, \theta_{n}\right),
$$

where the weight function is given by

$$
G(\theta)=e^{-2 m \tau_{0} \cosh \theta} K(\theta) .
$$

The key idea of [3] is that in the $t \rightarrow \infty$ limit only those contributions survive which do not depend on $t$. In our Euclidean setting this means, that the relevant terms do not depend on $x$. If we were able to derive the complete spectral expansion for $\langle O(x)\rangle_{R}^{\alpha \beta}$, then in principle it would be possible to reproduce (3.42) by collecting those terms which are completely independent of $x$. However, we can already make a definite statement about the $n=1$ term in (3.42). It is easy to see that up to higher order contributions this term is equal to our $J_{22}$ given by (3.38), which is indeed independent of $x$. This way we have established the first rigorous support for the series (3.42). ${ }^{6}$ However, at present we can not make any statements about the higher order terms. In particular only a detailed study of the higher order terms in $\langle O(x)\rangle_{R}^{\alpha \beta}$ can decide whether some kind of a "dressing" of $G(\theta)$ is needed to obtain the correct result, as it was suggested in [3].

\subsubsection{Relation to finite temperature correlation functions}

There is a way to connect the vacuum expectation values considered in this work to the evaluation of thermal correlation functions. The relation is not physical in the sense that it can be established only on a formal level. However, it is certainly worthwhile to explore this correspondence. For simplicity we suppose $g_{\alpha}=g_{\beta}=0$. Let us define a (non-local) operator $\mathcal{B}$ by specifying its form factors:

$$
\begin{aligned}
& \left\langle\theta_{1}, \theta_{2}, \ldots, \theta_{2 n-1}, \theta_{2 n}|\mathcal{B}| \theta_{1}^{\prime}, \theta_{2}^{\prime}, \ldots, \theta_{2 m-1}^{\prime}, \theta_{2 m}^{\prime}\right\rangle \equiv \\
& \equiv\left(\delta\left(\theta_{1}+\theta_{2}\right) K_{\alpha}\left(-\theta_{1}\right)\right) \ldots\left(\delta\left(\theta_{2 n-1}+\theta_{2 n}\right) K_{\alpha}\left(-\theta_{2 n-1}\right)\right) \\
& \quad \times\left(\delta\left(\theta_{1}^{\prime}+\theta_{2}^{\prime}\right) K_{\beta}\left(\theta_{1}^{\prime}\right)\right) \ldots\left(\delta\left(\theta_{2 m-1}^{\prime}+\theta_{2 m}^{\prime}\right) K_{\beta}\left(\theta_{2 m-1}^{\prime}\right)\right)+(\ldots) .
\end{aligned}
$$

where the dots in parentheses stand for disconnected terms which appear if two pairs $\left(\theta_{2 i-1}, \theta_{2 i}\right)$ and $\left(\theta_{2 j-1}^{\prime}, \theta_{2 j}^{\prime}\right)$ coincide for some $i=1, \ldots, n$ and $j=1, \ldots, m$. The form

\footnotetext{
${ }^{6}$ From a different point of view, the presence of the term $J_{22}$ in (3.42) supports our claim that the series (3.39) (which does not include $J_{22}$ ) can not be complete.
} 
factors with an odd number of particles on either sides are identically zero. Note that this definition of $\mathcal{B}$ incorporates information about both boundaries; actually the operator itself can be visualized by gluing the two boundaries together, thus forming a cylinder.

With this definition of $\mathcal{B}$ the vacuum expectation value $\langle O(x)\rangle_{R}^{\alpha \beta}$ can be expressed on a formal level as a two-point function at finite temperature $T=1 / R$ :

$$
\langle O(x)\rangle_{R}^{\alpha \beta} \equiv\langle\mathcal{B}(0) O(x)\rangle_{R}^{\text {periodic }} .
$$

This correspondence implies, that higher order terms in the v.e.v. $\left(D_{i j}\right.$ with $\left.i+j>4\right)$ will present technical difficulties which are very similar to those encountered in the evaluation of the thermal two-point function [7, 9, 17]. However, the exact relation between the two objects can only be established if the disconnected terms in the form factors of $\mathcal{B}$ are properly defined, ${ }^{7}$ which is out of the scope of the present work.

\section{Comparison with TCSA}

In this section we compare for various cases our spectral expansion (3.3) computed in the previous section with the numerical results of the truncated conformal space approach (TCSA). We choose the scaling Lee-Yang model, because despite being one of the simplest integrable models it already features many properties that we have discussed so far: it contains a single massive particle, it has tunable integrable boundary conditions with different $g_{j}$ values and all the form factors of its elementary field are known. Moreover, the TCSA is quite convergent for this model, which together with the properties listed above makes it an ideal testing ground for our method. In the first subsection we define the model and collect the relevant formulae which we will use to make the comparison in the second subsection.

\subsection{The boundary scaling Lee-Yang model}

In this section we summarise the relevant properties of the scaling Lee-Yang model described as a perturbed conformal field theory. We follow closely the treatment and notations of [13].

\subsubsection{The critical Lee-Yang model as a minimal conformal field theory}

The Lee-Yang model [39] is the simplest example of a non-unitary conformal field theory. It corresponds to the $M_{2,5}$ minimal model with central charge $-22 / 5$ and effective central charge 2/5. It has only two highest weight representations of the Virasoro algebra of weights 0 and $-1 / 5$, so there are only two bulk primary fields, the identity $\mathbb{1}$ of weight 0 and $\varphi$ of weight $-2 / 5$.

The conformal bounary conditions of the model were classified by Cardy in [40] and the field content on each of these is given by solving the consistency conditions written in $[41,42]$. The model has only two conformally invariant boundary conditions, denoted by $\mathbb{1}$ and $\Phi$. There is only one non-trivial boundary operator $\phi \equiv \phi_{-1 / 5}^{(\Phi, \Phi)}$ that can

\footnotetext{
${ }^{7} \mathrm{~A}$ naive substitution of the form factors of $\mathcal{B}$ without any disconnected terms results in a series similar to (3.39). This way one misses the diagonal contributions like $J_{22}$.
} 
live on the $\Phi$ boundary and there are two boundary changing operators, $\psi \equiv \phi_{-1 / 5}^{(11, \Phi)}$ and $\psi^{\dagger} \equiv \phi_{-1 / 5}^{(\Phi, 11)}$, which interpolate between the boundary conditions $\mathbb{1}$ and $\Phi$. These three boundary fields all have weight $h_{\phi}=h_{\psi}=1 / 5$.

For the sake of completeness we list all the operator product expansions (OPEs) and structure constants of the theory. The bulk OPE is

$$
\varphi(z, \bar{z}) \varphi(w, \bar{w})=C_{\varphi \varphi}{ }^{\mathbb{1}}|z-w|^{4 / 5}+C_{\varphi \varphi}^{\varphi}|z-w|^{2 / 5} \varphi(w, \bar{w})+\ldots,
$$

the boundary OPEs are

$$
\begin{aligned}
\phi(z) \phi(w) & =C_{\phi \phi} \mathbb{1}^{1}|z-w|^{2 / 5}+C_{\phi \phi}{ }^{\phi}|z-w|^{1 / 5} \phi(w)+\ldots, \\
\psi(z) \phi(w) & =C_{\psi \phi^{\dagger}}|z-w|^{1 / 5} \psi(w)+\ldots, \\
\phi(z) \psi^{\dagger}(w) & =C_{\phi \psi^{\dagger}} \psi^{\dagger}|z-w|^{1 / 5} \psi^{\dagger}(w)+\ldots, \\
\psi(z) \psi^{\dagger}(w) & =C_{\psi \psi^{\dagger}} \mathbb{1}^{\dagger}|z-w|^{2 / 5}+\ldots, \\
\psi^{\dagger}(z) \psi(w) & =C_{\psi^{\dagger} \psi}{ }^{\mathbb{1}}|z-w|^{2 / 5} q ;+C_{\psi^{\dagger} \psi}{ }^{\phi}|z-w|^{1 / 5} \phi(w)+\ldots,
\end{aligned}
$$

and the two bulk-boundary OPEs read

$$
\begin{aligned}
& \left.\varphi(z)\right|_{\mathbb{1}}={ }^{(\mathbb{1})} B_{\varphi}^{\mathbb{1}}|2(z-w)|^{2 / 5}+\ldots \\
& \left.\varphi(z)\right|_{\Phi}={ }^{(\Phi)} B_{\varphi}^{\mathbb{1}}|2(z-w)|^{2 / 5}+{ }^{(\Phi)} B_{\varphi}^{\phi}|2(z-w)|^{1 / 5} \phi(w)+\ldots .
\end{aligned}
$$

The structure constants can be chosen as [43]

$$
\begin{array}{rlrl}
C_{\varphi \varphi} \mathbb{1}=C_{\phi \phi} & =-1, & C_{\psi \psi^{\dagger}}{ }^{\mathbb{1}}=1, & C_{\psi^{\dagger} \psi} \mathbb{1}=-\frac{1+\sqrt{5}}{2}, \\
C_{\varphi \varphi} \varphi & =-\left|\frac{2}{1+\sqrt{5}}\right|^{1 / 2} \cdot \alpha^{2}, & { }^{(\mathbb{1})} B_{\varphi}^{\mathbb{1}}=-\left|\frac{2}{1+\sqrt{5}}\right|^{1 / 2}, \\
C_{\psi^{\dagger} \psi^{\phi}}=C_{\phi \phi}{ }^{\phi} & =-\left|\frac{1+\sqrt{5}}{2}\right|^{1 / 2} \cdot \alpha, & { }^{(\Phi)} B_{\varphi}^{\mathbb{1}}=\left|\frac{1+\sqrt{5}}{2}\right|^{3 / 2}, \\
C_{\phi \psi^{\dagger}} \psi^{\dagger}=C_{\psi \phi}{ }^{\psi} & =-\left|\frac{2}{1+\sqrt{5}}\right|^{1 / 2} \cdot \alpha, & { }^{(\Phi)} B_{\varphi}^{\phi}=\left|\frac{5+\sqrt{5}}{2}\right|^{1 / 2} \cdot \alpha, \\
\alpha & =\left|\frac{\Gamma(1 / 5) \Gamma(6 / 5)}{\Gamma(3 / 5) \Gamma(4 / 5)}\right|^{1 / 2} . &
\end{array}
$$

We will need the conformal expectation value of the bulk field $\varphi$ across the strip of width $R$ with various combinations of boundary conditions: $(\mathbb{1}, \mathbb{1}),(\Phi, \mathbb{1})$ and $(\Phi, \Phi)$. Our coordinates are $0 \leq x \leq R$ across the strip and $y$ running along the strip, and we normalise all our correlation functions so that the expectation value of the identity operator is always one.

The correlation functions on the strip with boundary conditions $\alpha$ and $\beta$ can be determined by mapping the strip to the unit disc or the upper half plane and inserting the appropriate boundary fields $\phi_{-1 / 5}^{\alpha \beta}$. Under the exponential map $z=\exp (i \pi(x-i y) / R$ the strip can be mapped to the upper half plane where the boundaries are mapped onto the negative and positive real axis. The boundaries meet at the origin (and at infinity) where 
boundary operators are inserted according to the boundary conditions. Through radial quantisation the Hilbert space of the theory is built on the representations corresponding to the boundary operators at the origin. ${ }^{8}$ It follows that for the $(\mathbb{1}, \mathbb{1})$ boundaries the Hilbert space consists of the $\mathbb{1}$ module, in the $(\mathbb{1}, \Phi)$ case of the $\Phi$ module and for $(\Phi, \Phi)$ both modules are present with the ground state corresponding to $\phi$.

If at least one of the boundaries is the $\Phi$ then the boundary field $\phi(y)$ may exist on it. For these cases the boundary one-point functions are

$$
\langle\phi(y)\rangle_{(\Phi, \mathbb{1})}=\left(\frac{R}{\pi}\right)^{1 / 5} C_{\psi \phi} \psi, \quad\langle\phi(y)\rangle_{(\Phi, \Phi)}=\left(\frac{R}{\pi}\right)^{1 / 5} C_{\phi \phi}{ }^{\phi} .
$$

The one-point functions of the bulk field $\varphi(x)$ on the strip correspond in general to chiral three-point functions on the upper half plane, and by the doubling trick they can be thought of as four-point functions on the full complex plane. Since the representation $\phi$ has a null-vector at the second level, the correlation functions satisfy certain differential equations, the solutions of which can be expressed in terms of the four strip chiral block functions

$$
\begin{aligned}
& f_{1}(\vartheta)=\left(\frac{2 \sin \vartheta}{\cos ^{2} \vartheta}\right)^{2 / 5}{ }_{2} \mathrm{~F}_{1}\left(\frac{4}{10}, \frac{9}{10} ; \frac{11}{10} ;-\tan ^{2} \vartheta\right), \\
& f_{2}(\vartheta)=\left(\frac{2 \sin \vartheta}{\cos ^{3} \vartheta}\right)^{1 / 5}{ }_{2} \mathrm{~F}_{1}\left(\frac{3}{10}, \frac{8}{10} ; \frac{9}{10} ;-\tan ^{2} \vartheta\right), \\
& f_{3}(\vartheta)=(2 \sin \vartheta)^{2 / 5}, \\
& f_{4}(\vartheta)=(2 \sin \vartheta)^{1 / 5},
\end{aligned}
$$

where $\vartheta=x \pi / R$. The particular solutions are fixed by the boundary conditions, that is the corresponding bulk-boundary OPEs:

$$
\begin{aligned}
\langle\varphi(x, y)\rangle_{(\mathbb{1}, \mathbb{1})} & =\left(\frac{R}{\pi}\right)^{2 / 5}{ }^{(\mathbb{1})} B_{\varphi}^{\mathbb{1}} f_{3}\left(\frac{\pi x}{R}\right) ;={ }^{(\mathbb{1})} B_{\varphi}^{\mathbb{1}}\left(\frac{2 R}{\pi} \sin \frac{\pi x}{R}\right)^{2 / 5}, \\
\langle\varphi(x, y)\rangle_{(\Phi, \Phi)} & =\left(\frac{R}{\pi}\right)^{2 / 5}\left({ }^{(\Phi)} B_{\varphi}^{\mathbb{1}} f_{1}\left(\frac{\pi x}{R}\right)+{ }^{(\Phi)} B_{\varphi}^{\phi} C_{\phi \phi}{ }^{\phi} f_{2}\left(\frac{\pi x}{R}\right)\right), \\
\langle\varphi(x, y)\rangle_{(\Phi, \mathbb{1})} & =\left(\frac{R}{\pi}\right)^{2 / 5} \begin{cases}{ }^{(\Phi)} B_{\varphi}^{\mathbb{1}} f_{1}\left(\frac{\pi x}{R}\right)+{ }^{(\Phi)} B_{\varphi}^{\phi} C_{\psi \phi} \psi f_{2}\left(\frac{\pi x}{R}\right), & x \leq \frac{R}{2}, \\
{ }^{(\mathbb{1})} B_{\varphi}^{\mathbb{1}} f_{1}\left(\frac{\pi x}{R}\right), & x>\frac{R}{2} .\end{cases}
\end{aligned}
$$

The latter is the unique combination of the chiral blocks with the proper asymptotic behaviour near the boundaries, for $\vartheta \rightarrow 0$ and $\vartheta \rightarrow \pi$.

Since for the $(\Phi, \Phi)$ boundary conditions both representations are present in the Hilbert

\footnotetext{
${ }^{8}$ Thus the states on the strip, unlike the bulk case, are characterised by their behaviour under one copy of the Virasoro algebra.
} 
space, we will need also the non-diagonal matrix elements

$$
\begin{aligned}
& \langle\phi|\varphi(x, y)| \mathbb{1}\rangle=\left(\frac{R}{\pi}\right)^{1 / 5}{ }^{(\Phi)} B_{\varphi}^{\phi} f_{4}\left(\frac{\pi x}{R}\right)={ }^{(\Phi)} B_{\varphi}^{\phi}\left(\frac{2 R}{\pi} \sin \frac{\pi x}{R}\right)^{1 / 5}, \\
& \langle\mathbb{1}|\varphi(x, y)| \phi\rangle=-\left(\frac{R}{\pi}\right)^{1 / 5}{ }^{(\Phi)} B_{\varphi}^{\phi} f_{3}\left(\frac{\pi x}{R}\right)=-{ }^{(\Phi)} B_{\varphi}^{\phi}\left(\frac{2 R}{\pi} \sin \frac{\pi x}{R}\right)^{2 / 5} .
\end{aligned}
$$

Due to translational invariance in the "time" direction none of the expectation values depends on $y$.

\subsubsection{The scaling Lee-Yang model}

The scaling Lee-Yang (SLY) model can be defined as a bulk perturbation of the critical Lee-Yang model by the term

$$
\lambda \int \mathrm{d} x \int \mathrm{d} y \varphi(x, y) .
$$

The resulting theory is an integrable massive scattering theory, containing a single particle with two-particle S-matrix [44]

$$
S(\theta)=-(1)(2), \quad(x)=\frac{\sinh \left(\frac{\theta}{2}+\frac{i \pi x}{6}\right)}{\sinh \left(\frac{\theta}{2}-\frac{i \pi x}{6}\right)} .
$$

The mass $M$ of the particle is related to the strength $\lambda$ of the perturbation by $[45,46]$

$$
M=\kappa \lambda^{5 / 12}, \quad \kappa=2^{19 / 12} \sqrt{\pi} \frac{(\Gamma(3 / 5) \Gamma(4 / 5))^{5 / 12}}{5^{5 / 16} \Gamma(2 / 3) \Gamma(5 / 6)}=2.642944 \ldots
$$

The form factors of the bulk field $\varphi$ were first computed in [47, 48], but following [13] we use the conventions of [49], with the difference that for us $\varphi(x)$ is a real field. The function $F_{n}$ can be parametrised as

$$
F_{n}\left(\theta_{1}, \ldots, \theta_{n}\right)=H_{n} Q_{n}\left(x_{1}, \ldots, x_{n}\right) \prod_{i<j}^{n} \frac{f\left(\theta_{i}-\theta_{j}\right)}{x_{i}+x_{j}},
$$

where $x_{i}=e^{\theta_{i}}$. The various terms in (4.10) are

$$
f(\theta)=\frac{\cosh \theta-1}{\cosh \theta+1 / 2} v(i \pi-\theta) v(-i \pi+\theta)
$$

where $v$ is given by $^{9}$

$$
\begin{aligned}
v(\theta)= & \prod_{n=1}^{N}\left[\frac{\left(\frac{\theta}{2 \pi i}+n+1 / 2\right)\left(\frac{\theta}{2 \pi i}+n-1 / 6\right)\left(\frac{\theta}{2 \pi i}+n-1 / 3\right)}{\left(\frac{\theta}{2 \pi i}+n-1 / 2\right)\left(\frac{\theta}{2 \pi i}+n+1 / 6\right)\left(\frac{\theta}{2 \pi i}+n+1 / 3\right)}\right]^{n} \\
& \times \exp \left(2 \int_{0}^{\infty} \mathrm{d} t \frac{\sinh (t / 2) \sinh (t / 3) \sinh (t / 6)}{t \sinh ^{2}(t)}\left(N+1-N \mathrm{e}^{-2 t}\right) \mathrm{e}^{-2 N t+i \theta t / \pi}\right), \\
v(0)= & 1.111544045 \ldots,
\end{aligned}
$$

\footnotetext{
${ }^{9}$ This form with the finite product in front improves the convergence of the integral [49].
} 
and the normalisation factor is [50]

$$
H_{n}=\psi\left(-\frac{i 3^{1 / 4}}{\sqrt{2} v(0)}\right)^{n}
$$

Here $\psi$ is the expectation value $\langle\varphi\rangle$ in the bulk:

$$
\psi=\frac{-3^{\frac{9}{10}} \Gamma\left(\frac{1}{3}\right)^{\frac{36}{5}} M^{-\frac{2}{5}}}{(2 \pi)^{\frac{14}{5}} 5^{\frac{1}{4}} \Gamma\left(\frac{1}{5}\right) \Gamma\left(\frac{2}{5}\right)}=(-1.239394325 \ldots) M^{-2 / 5} .
$$

The symmetric polynomials $Q_{n}\left(x_{1}, \ldots, x_{n}\right)$ have degree $n(n-1) / 2$ and partial degree $n-1$. They can be neatly expressed as a determinant of a matrix in symmetric polynomials [47-49]. Here we will need only the explicit form of the first few:

$$
Q_{0}=1, \quad Q_{1}=1, \quad Q_{2}=\sigma_{1}^{(2)}, Q_{3}=\sigma_{2}^{(3)} \sigma_{1}^{(3)}, Q_{4}=\sigma_{3}^{(4)} \sigma_{2}^{(4)} \sigma_{1}^{(4)}
$$

where the elementary symmetric polynomials in $n$ variables $\sigma_{r}^{(n)}$ are defined by

$$
\prod_{i=1}^{n}\left(1+p x_{i}\right)=\sum_{k=0}^{n} p^{k} \sigma_{k}^{(n)}
$$

Let us turn now to the boundary conditions for the SLY model which were analysed in detail in [35]. The integrable boundary conditions are the $\mathbb{1}$ conformal boundary condition and the perturbation of the conformal $\Phi$ boundary by

$$
h \int \phi(x) \mathrm{d} x
$$

which we will denote by $\Phi(h)$. The exact reflection factors for these two boundary conditions are

$$
R_{\Phi(h)}(\theta)=R_{b}(\theta), \quad R_{\mathbb{1}}(\theta)=R_{0}(\theta)
$$

where

$$
R_{b}(\theta)=\left(\frac{1}{2}\right)\left(\frac{3}{2}\right)\left(\frac{4}{2}\right)^{-1}\left(S\left(\theta+i \pi \frac{b+3}{6}\right) S\left(\theta-i \pi \frac{b+3}{6}\right)\right)^{-1} .
$$

The relation between $b$ and $h$ is given by [35]

$$
h(b)=-\left|\hat{h}_{c}\right| \sin (\pi(b+1 / 2) / 5) .
$$

For numerical calculations it is more suitable to use instead the dimensionless quantity

$$
\hat{h}_{\text {crit }}=\hat{h}_{c} M^{-6 / 5},
$$

which was determined in [51]:

$$
\hat{h}_{\text {crit }}=-\pi^{3 / 5} 2^{4 / 5} 5^{1 / 4} \frac{\sin \frac{2 \pi}{5}}{\left(\Gamma\left(\frac{3}{5}\right) \Gamma\left(\frac{4}{5}\right)\right)^{1 / 2}}\left(\frac{\Gamma\left(\frac{2}{3}\right)}{\Gamma\left(\frac{1}{6}\right)}\right)^{6 / 5}=-0.68528998399118 \ldots .
$$


Finally, let us consider the boundary-particle couplings $g_{\alpha}$ for the various boundary conditions. We recall that these quantities are defined via the residue of the reflection factor $R_{\alpha}(\theta)$ at the rapidity $\theta=i \pi / 2$

$$
R_{\alpha}(\theta) \sim \frac{i}{2} \frac{\left(g_{\alpha}\right)^{2}}{\theta-i \pi / 2}
$$

In the SLY model we have

$$
g_{\mathbb{1}}=-i 2 \sqrt{2 \sqrt{3}-3}
$$

for the $\mathbb{1}$ boundary, and

$$
g_{\Phi}(b)=\frac{\tan ((b+2) \pi / 12)}{\tan ((b-2) \pi / 12)} g_{\Perp}
$$

for the $\Phi(h(b))$ boundary. Notice that although the reflection factors for the boundaries $\mathbb{1}$ and $\Phi(h(0))$ are identical, the corresponding boundary-particle couplings differ by a sign. This means that the boundary states for these two boundary conditions differ only in the sign of the contributions from states of odd particle number.

\subsection{The comparison}

\subsubsection{Expectation values in the truncated conformal space approach}

The TCSA method allows for calculating the spectrum and other physical quantities of a perturbed conformal field theory. The approach was developed in [52] for the bulk scaling Lee-Yang model itself and later it was generalised to systems with boundary [35, 51]. The idea of the method is very simple: using the conformal field theory techniques one can calculate in the conformal basis all the matrix elements of the perturbing operator(s) and eventually of the perturbed Hamiltonian. If the conformal Hilbert space is truncated at some energy (or at some conformal level), the space of states becomes finite dimensional and the calculation of the spectrum and of the eigenvectors of the perturbed Hamiltonian boils down to the diagonalisation of a finite numerical matrix. This approach can be regarded as a close relative of the standard variational method, and although the errors caused by the truncation can not be easily controlled, it is generally believed, and checked of course, that they decrease with increasing the cut. We refer the reader interested in the details of the behaviour of TCSA to $[53,54]$.

The Hamiltonian of the boundary scaling Lee-Yang model reads

$$
H\left(R ; \lambda, h_{l}, h_{r}\right)=H_{0}+\lambda \int_{0}^{R} \varphi(x, y) \mathrm{d} x+h_{l} \phi(x=0)+h_{r} \phi(x=R),
$$

where $H_{0}$ is the conformal Hamiltonian and we have allowed for boundary perturbations at the edges of the strip (they can be present only for $\Phi$ boundaries). The matrix elements of the Hamiltonian, calculated by mapping the strip on the upper half plane are given by

$h_{i j}=\frac{\pi}{r}\left(\left(h_{i}-\frac{c}{24}\right) \delta_{i j}+\kappa^{\prime}\left(\frac{r}{\pi}\right)^{12 / 5}\left(G^{-1} B\right)_{i j}+\chi_{l}\left(\frac{r}{\pi}\right)^{6 / 5}\left(G^{-1} B_{l}\right)_{i j}+\chi_{r}\left(\frac{r}{\pi}\right)^{6 / 5}\left(G^{-1} B_{r}\right)_{i j}\right)$, 


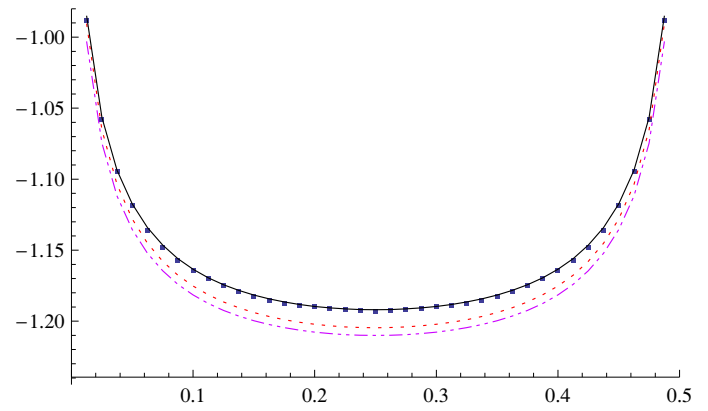

(a) $(\Phi(-2), \Phi(-2))$ at $r=0.5$

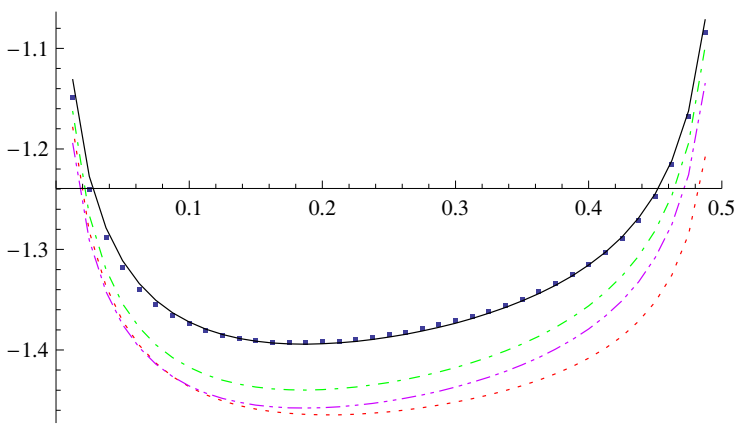

(b) $(\Phi(-1 / 2), \Phi(-2))$ at $r=0.5$

Figure 2. $\left\langle M^{2 / 5} \varphi(x)\right\rangle$ vs. $\xi \in(0 \ldots r)$. The dots are TCSA results with cut $N=10$ (48 states), the green dot-dashed and the solid black line is our result (4.31) up to the 3rd and 4th orders, respectively. The dotted red line is (4.33) and the violet dot-dot-dashed line is (4.31) without the term $J_{22}$. The horizontal axes are positioned at the conformal $\langle\varphi\rangle_{0}$.

where

$$
\begin{aligned}
\left(B_{l}\right)_{i j} & =\langle i|\phi(1)| j\rangle, & \left(B_{r}\right)_{i j}=\langle i|\phi(-1)| j\rangle, \\
(B)_{i j} & =\left\langle i\left|\int_{0}^{\pi} \mathrm{d} \vartheta \varphi\left(e^{i \vartheta}\right)\right| j\right\rangle, &
\end{aligned}
$$

and the conformal metric $G$ defined as $G_{i j}=\langle i \mid j\rangle$ is needed because the basis vectors are not orthonormal. Here every operator is understood to be on the upper half plane, $\vartheta=\pi x / R$ and we expressed everything in dimensionless form:

$$
\begin{aligned}
r & =M R, & \kappa^{\prime} & =\kappa^{12 / 5}=0.097048456298 \ldots, \\
\chi_{l} & =\sin \left(\pi\left(b_{l}+1 / 2\right) / 5\right) \hat{h}_{\text {crit }}, & \chi_{r} & =\sin \left(\pi\left(b_{r}+1 / 2\right) / 5\right) \hat{h}_{\text {crit }},
\end{aligned}
$$

implying that the energy eigenvalues are also measured in the units of $M$.

Once the spectrum is known the expectation value of $\varphi$ can be estimated by

$$
\left\langle M^{2 / 5} \varphi(x)\right\rangle \sim\left(\frac{r}{\pi}\right)^{2 / 5} \frac{\langle\Omega|\varphi(\exp (i \pi \xi / r))| \Omega\rangle}{\langle\Omega \mid \Omega\rangle},
$$

where $\xi=M x$ is the dimensionless position of the operator on the strip and $|\Omega\rangle$ is the ground state eigenvector. If we know the matrix elements of $\varphi(\vartheta)$ on the upper half plane, the calculation of the v.e.v. amounts to matrix-vector multiplication.

An efficient way to calculate the matrix elements is given in the appendix of [55].

\subsubsection{Comparing the form factor expansion with TCSA}

Let us recall our main result that we will compare with the TCSA data:

$$
\langle O(x)\rangle_{R}^{\alpha \beta}=\langle O\rangle+\sum_{i+j \leq 4} D_{i j}+\ldots .
$$

In the following we will refer to terms $D_{i j}$ with $i+j=n$ as $n$th order terms. 


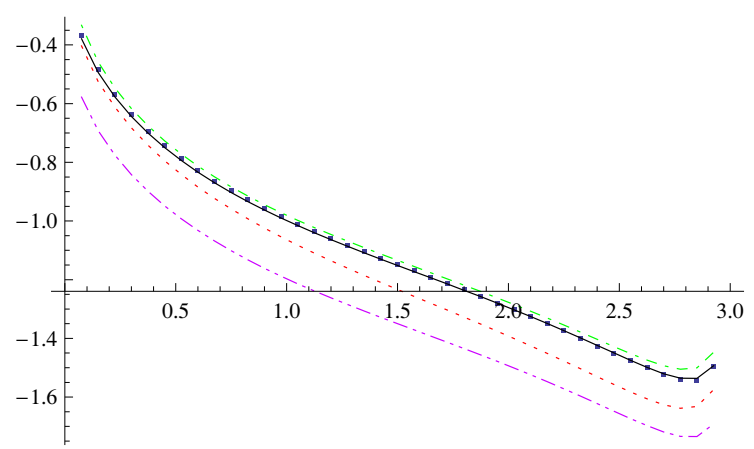

(a) $(\mathbb{1}, \Phi(0))$ at $r=3$

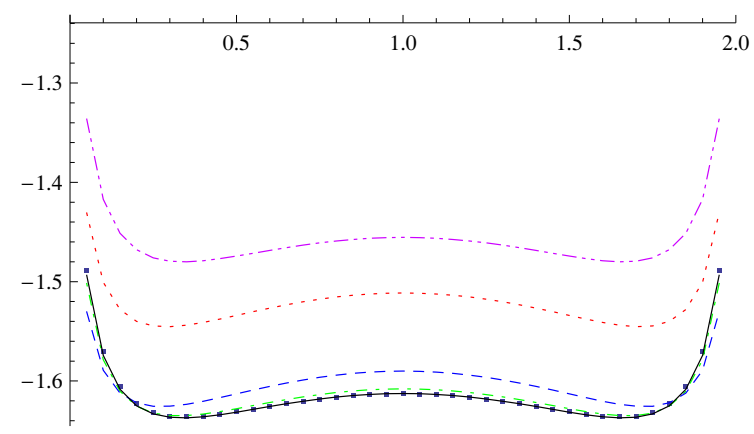

(b) $(\Phi(-1 / 2), \Phi(-1 / 2))$ at $r=2$

Figure 3. $\left\langle M^{2 / 5} \varphi(x)\right\rangle$ vs. $\xi \in(0 \ldots r)$. The dots are TCSA results with cut $N=12$ (45 states) in (a) and $N=10$ (48 states) in (b), the blue dashed, the green dot-dashed and the solid black line is our result (4.31) up to the 2nd, 3rd and 4th orders, respectively. The dotted red line is (4.33) and the violet dot-dot-dashed line is $(4.31)$ with the sign of the term $D_{11}$ flipped. The horizontal axes are positioned at the conformal $\langle\varphi\rangle_{0}$.

The work [13] addressed the calculation of the vacuum expectation value in large volume when the operator is close to one of the boundaries. With this setup they could neglect the effect of the other boundary and they worked up to the third order, so their formula in our notations is

$$
\langle O\rangle+D_{10}+D_{20}+D_{30}
$$

One can try to improve this result for finite strips by considering also the analogous terms coming from the other boundary:

$$
\langle O\rangle+\sum_{i=1}^{3}\left(D_{i 0}+D_{0 i}\right) .
$$

In order to show that our formula means a great improvement we will compare this expression with our result (4.31).

Figure 2(a) shows the TCSA data with cut $N=10$ (48 states) together with our results for $\left\langle M^{2 / 5} \varphi(\xi)\right\rangle$ for the boundary conditions $(\Phi(b=-2), \Phi(b=-2))$. In this case $g_{\alpha}=g_{\beta}=0$ and only the even orders are non-zero. In particular, in this special case formula (4.33) coincides with our second order result. In figure 2(b) we plot the case of the boundaries $(\Phi(b=-1 / 2), \Phi(b=-2))$. The TCSA has already converged, since changing the cut from $N=9$ (38 states) to $N=10$ (48 states) the maximum change in the v.e.v. is $\approx 0.0005$. The plots clearly show that our formula gives much better results than (4.33) throughout the strip. We also plotted our result without the term $J_{22}$ (violet dot-dotdashed lines) in order to show that this non-trivial term is indeed present in the expansion (see the discussion in section 3.4.1).

Figure 3 shows the v.e.v. for other pairs of boundary conditions. For the second pair changing the TCSA cut from $N=9$ to $N=10$ resuls in a change of order $10^{-5}$ in the v.e.v. For the $(\mathbb{1}, \Phi(0))$ boundaries raising the cut from $N=8$ (18 states) to $N=12$ (45 states) the change in the v.e.v. is only $\approx 0.05$. 
In order to show that the conventions for the complex conjugation of the $g$ factors are correct we plot also the result with the sign of the term $D_{11}$ reversed (violet dashed lines). In figure 3(b) we show also the second order result to show the convergence of the form factor expansion. It is clearly seen in both plots that adding the fourth order terms hardly changes the result, thus in these cases the form factor series is almost saturated by its first four orders.

In figure 4 we plot the one-point function for the $(\mathbb{1}, \mathbb{1})$ boundaries for different strip widths. The TCSA is very convergent also in this case: a change in the cut from $N=8$ (12 states) to $N=12$ (29 states) causes a shift of order $10^{-5}$ in the v.e.v. The convergence of the form factor expansion is again obvious, however, for $r=2$ our formula starts to deviate from the TCSA values, as a clear sign of the need for higher order terms. Our result is closer to the real values than the formula (4.33), but what is surprising is that the result of [13], expression (4.32), performs very well in the vicinity of the boundary. This can be understood by looking at the leading behaviour of the expectation value near the different boundaries [13]:

$$
\begin{aligned}
\left\langle M^{2 / 5} \varphi(x)\right\rangle_{\Phi(0)} & =(2 \xi)^{1 / 5(\Phi)} B_{\varphi}^{\phi}\langle\phi\rangle_{\Phi(0)}+(2 \xi)^{2 / 5(\Phi)} B_{\varphi}^{\mathbb{1}}\langle\mathbb{1}\rangle_{\Phi(0)}+\mathcal{O}\left(\xi^{12 / 5}\right), \\
\left\langle M^{2 / 5} \varphi(x)\right\rangle_{\mathbb{1}} & =(2 \xi)^{2 / 5(\mathbb{1})} B_{\varphi}^{\mathbb{1}}\langle\mathbb{1}\rangle_{\mathbb{1}}+\mathcal{O}\left(\xi^{12 / 5}\right)
\end{aligned}
$$

We have to check what happens when $x$ is fixed and the volume $R$ is changed. We know that the corrections to (4.32) are of the form $\exp (-M R)$, thus rescaling $R$ is equivalent to a rescaling of $M$. Equation (4.34b) remains unchanged under such a rescaling in the leading order (and the next term is two orders higher in $\xi$ ). In other words, the $x$-dependence of the v.e.v. agrees with the behaviour under a conformal rescaling, which means that for the $\mathbb{1}$ boundary the expectation value will not change much when the width of the strip changes. Obviously this is not true for (4.34a). ${ }^{10}$

Since our truncation of the form factor series is truly consistent only at $x=R / 2$, in figure 5 we plot the deviation of the v.e.v. from the TCSA values at the middle of the strip for different volumes $R$. It is clearly seen on the logarithmic plot that for large enough volume $(r \gtrsim 2)$ the errors decrease exponentially with the volume and the exponents for the error of the consecutive orders are approximately even-spaced. In particular, the sum of all four orders has the smallest and most rapidly decreasing error. A similar behaviour can be observed for other boundary conditions.

\section{Conclusions}

In this paper we considered vacuum expectation values of local operators in the presence of two integrable boundaries. We developed a consistent finite volume regularisation scheme to handle the various types of disconnected terms. The method presented in this work can be considered as a generalisation of the approach of [11] which dealt with expectation values with periodic boundary conditions. The key ingredients of our approach are the

\footnotetext{
${ }^{10}$ We would like to thank Gérard Watts for discussing this question.
} 


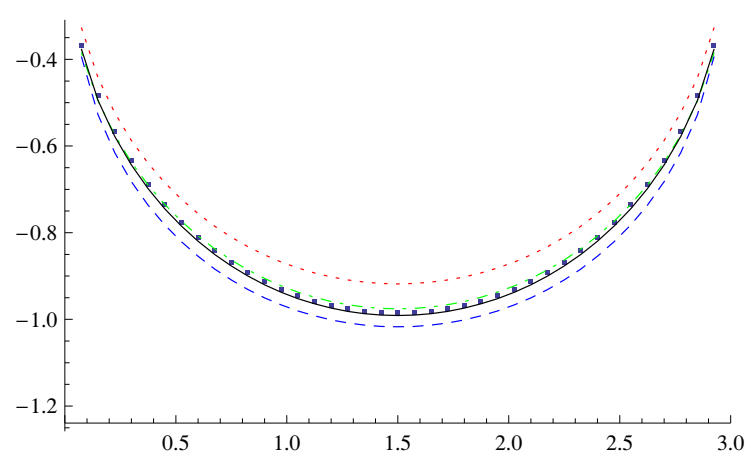

(a) $(\mathbb{1}, \mathbb{1})$ at $r=3$

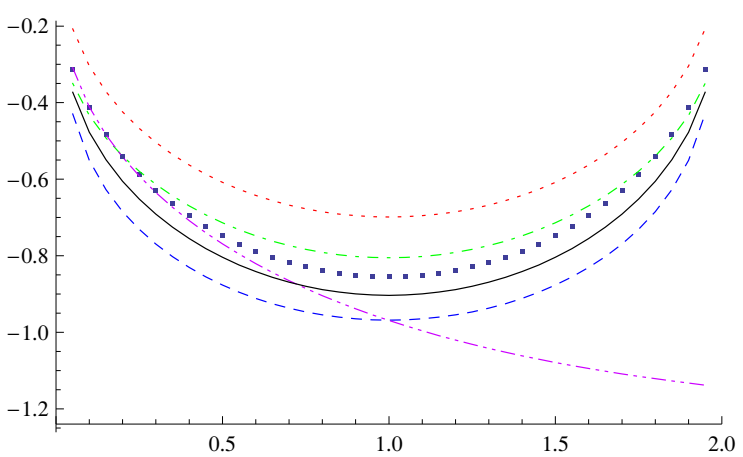

(b) $(\mathbb{1}, \mathbb{1})$ at $r=2$

Figure 4. $\left\langle M^{2 / 5} \varphi(x)\right\rangle$ vs $\xi \in(0 \ldots r)$. The dots are TCSA results with cut $N=12$ (29 states), the blue dashed, green dot-dashed and the solid black line is our result (4.31) up to the 2nd, 3rd and 4th orders, respectively. The dotted red line is (4.33) and the asymmetric violet dot-dot-dashed line in (b) is (4.32). The horizontal axes are positioned at the conformal $\langle\varphi\rangle_{0}$.

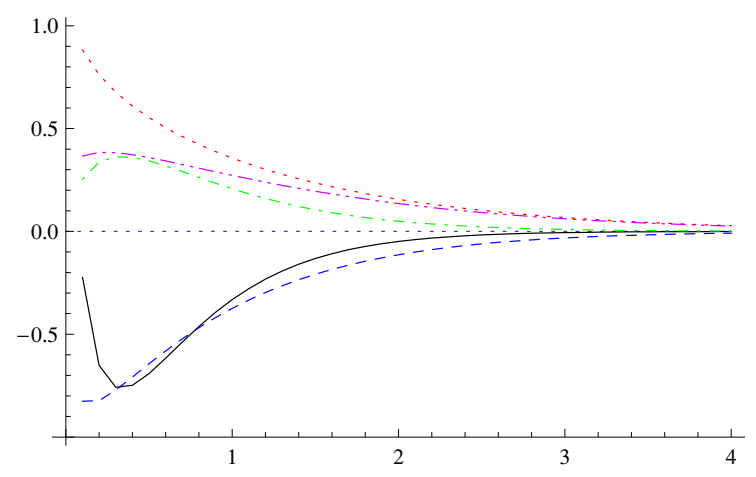

(a) linear scale

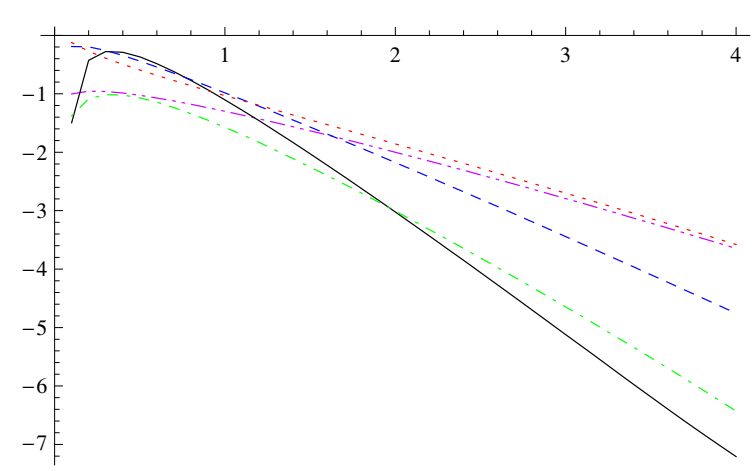

(b) $\log$ scale

Figure 5. $\left\langle M^{2 / 5} \varphi\left(x=\frac{R}{2}\right)\right\rangle-\left\langle M^{2 / 5} \varphi\left(\frac{R}{2}\right)\right\rangle_{\text {TCSA }}$ for b.c. (11, $\left.\mathbb{1}\right)$ with TCSA cut $N=12$ (29 states) as a function of $r$. The violet dot-dot-dashed, the blue dashed, the green dot-dashed and the black solid line is our result (4.31) up to the 1st, 2nd, 3rd and 4th order, respectively. The red dotted line is formula (4.33). For $r \gtrsim 2$ the errors decrease exponentially with $r$.

proper normalisation of the boundary state in a finite volume and the knowledge of the finite volume form factors.

Apart from the task of evaluating disconnected terms of the form factors the twoboundary setting poses additional difficulties for $g_{\alpha} g_{\beta} \neq 0$. In this case the boundary states include zero-momentum particles corresponding to the poles of the amplitudes $K_{j}(\theta)$ at $\theta=0$. We showed that our finite volume evaluation scheme automatically provides a regularisation of the resulting singularities. We developed a new method to extract the finite parts (see Theorem 1 in section 2.2) which can be applied also in other cases, e.g. two-point functions at finite temperature.

As a by-product of our formalism we were able to extract the second order terms of the ground state energy on the strip (subsection 2.2). This way we confirmed the 
boundary Thermodynamic Bethe Ansatz (BTBA) equations and we showed that they yield the correct second order terms even in the presence of zero-momentum particles $\left(g_{\alpha} g_{\beta} \neq 0\right)$, for which no rigorous derivation of the BTBA is known. Also, we gave further independent support for the normalisation condition $\bar{g}_{j}=g_{j} / 2$, which was proven by other means in $[19,20]$.

It is possible to compute higher order terms of the vacuum expectation value $\langle O(x)\rangle_{R}^{\alpha \beta}$ using the methods laid out in this work; in particular the finite volume normalisation of higher multi-particle contributions to the boundary state follows in a straightforward manner. However, the evaluation of the $L \rightarrow \infty$ limit poses technical difficulties already at the next orders $\left(D_{i j}\right.$ with $\left.i+j>4\right)$. There will appear new types of singularities which are very similar to those encountered in the evaluation of thermal correlation functions (see subsection 3.4.3). These difficulties call for new summation schemes to separate the divergent and finite parts of the relevant finite volume series. Alternatively, it would be interesting to develop a general infinite volume regularisation scheme along the lines of $[6,17]$, which could be compared to the results of the present approach.

There is plenty of room for future extensions of this work. First of all, one can consider expectation values with respect to a boundary excited state. In this case one has to develop an appropriate modification of the finite volume boundary state formalism. A further interesting problem is the evaluation of (space and time dependent) correlation functions on the strip, which can be performed along the lines of the present paper. Future work is needed to generalise our methods to non-diagonal scattering theories which are relevant to a number of condensed matter problems [56]. Also, it needs to be clarified whether some of the present results also apply to massless scattering theories. In the absence of a mass gap the convergence of the spectral series is not guaranteed anymore and one does not have control over residual finite size corrections to form factors. However, in some cases it is possible to apply a modified version of the form factor program to massless theories $[57,58]$. The study of a finite volume regularisation scheme for massless theories is left as an open problem.

\section{Acknowledgments}

We would like to thank Gábor Takács and Gérard Watts for useful discussions. The early stages of this work carried out while B. P. was a research assistant in the Theoretical Physics Group of the Hungarian Academy of Sciences. Also, he was partially supported by the grant OTKA K75172 of the HAS. M. K. was supported by the grants INSTANS (from ESF) and 2007JHLPEZ (from MIUR).

\section{A Regularisation of the divergent sums}

For a given $L$ let us denote by $\theta_{I}$ the solutions of the quantisation condition

$$
Q(\theta)=m L \sinh \theta+\delta(2 \theta)=2 \pi I, \quad I \in \mathbb{N}+\frac{1}{2} .
$$


where $\delta(\theta)$ is the elastic phase shift defined by

$$
S(\theta)=-e^{i \delta(\theta)}, \quad \delta(-\theta)=-\delta(\theta) .
$$

We also introduce the density of states

$$
\bar{\rho}_{1}(\theta)=\frac{d Q(\theta)}{d \theta}=m L \cosh \theta+2 \varphi(2 \theta),
$$

where $\varphi(\theta)=\delta^{\prime}(\theta)$.

Theorem 1: Let $f(\theta)$ be a symmetric function which apart from a double pole at $\theta=0$ is analytic in a neighbourhood of the real axis:

$$
f(\theta) \approx \frac{G}{\theta^{2}} \quad \text { as } \quad \theta \rightarrow 0 .
$$

Then the expression

$$
S(L)=\left(\sum_{I} \frac{f\left(\theta_{I}\right)}{\bar{\rho}_{1}\left(\theta_{I}\right)}\right)-\frac{G}{8} m L
$$

has a regular behaviour at large $L$ with the $L \rightarrow \infty$ limit given by

$$
\lim _{L \rightarrow \infty} S(L)=I_{f}+K_{f}
$$

where

$$
I_{f}=\int_{-\infty}^{\infty} \frac{d \theta}{4 \pi}\left(f(\theta)-G \frac{\cosh \theta}{\sinh ^{2} \theta}\right) \quad \text { and } \quad K_{f}=\frac{G}{4} \varphi(0) .
$$

Proof. We express the sum in the form of a complex integral for a finite $L$ and perform the $L \rightarrow \infty$ limit afterwards. First of all, the summation over the rapidities can be replaced by a sum over contour integrals around the solutions of $e^{i Q}+1=0$ :

$$
-\frac{1}{2} \sum \oint \frac{1}{2 \pi} \frac{f(\theta)}{e^{i Q}+1}
$$

where now the summation is over $I \in \mathbb{Z}+1 / 2$. The integration contours can be transformed into two distinct curves:

- the first starting from $\theta=\infty+i \varepsilon$ running to $\varepsilon+i \varepsilon$, crossing the real axis between 0 and the first solution of (A.1), then running from $\theta=\varepsilon-i \varepsilon$ to $\theta=\infty-i \varepsilon$;

- and a similar curve around the negative real axis, with the same counter-clockwise orientation.

These two curves can be joined to form a single contour encircling the whole real axis. In doing this, one picks up the residue at $\theta=0$, which is given by

$$
\frac{1}{2} \operatorname{Res}_{\theta=0} \frac{f(\theta)}{e^{i Q(\theta)}+1}=-i \frac{G}{8} \bar{\rho}_{1}(0)=-i \frac{G}{8}(m L+2 \varphi(0)) .
$$


Therefore the $\mathcal{O}(L)$ terms cancel and we obtain

$$
S(L)=\left(\int_{-\infty+i \varepsilon}^{\infty+i \varepsilon}-\int_{-\infty-i \varepsilon}^{\infty-i \varepsilon}\right) \frac{1}{4 \pi} \frac{f(\theta)}{e^{i Q}+1}+\frac{G}{4} \varphi(0) .
$$

Note that in the two integrals the $L$ dependence is only contained in $Q(\theta)$. We may now perform the $L \rightarrow \infty$ limit for a fixed $\varepsilon>0$. To do this first of all note that the integrand has become bounded (we stay away from the real axis) therefore one may exchange the limit $L \rightarrow \infty$ with the integration. Also, observe that

$$
\lim _{L \rightarrow \infty} \frac{1}{e^{i Q(\theta+i \varepsilon)}+1}=1, \quad \quad \lim _{L \rightarrow \infty} \frac{1}{e^{i Q(\theta-i \varepsilon)}+1}=0 .
$$

Putting everything together one obtains

$$
\int_{-\infty+i \varepsilon}^{\infty+i \varepsilon} \frac{1}{4 \pi} f(\theta)+\frac{G}{4} \varphi(0) .
$$

The integral above can be pulled back to the real axis after an appropriate regularisation. We use the identity

$$
0=\int_{-\infty+i \varepsilon}^{\infty+i \varepsilon} \frac{1}{4 \pi} G \frac{\cosh \theta}{\sinh ^{2} \theta}
$$

which can be proven by deforming the contour to $\operatorname{Im} \theta=i \pi / 2$. Moreover, the function above has exactly the same singularity structure around $\theta=0$ as the integrand in (A.3). One may therefore subtract (A.4) from (A.3) and pull back the integration contour to the real axis. The final result is thus given by

$$
\lim _{L \rightarrow \infty} S(L)=\int_{-\infty}^{\infty} \frac{d \theta}{4 \pi}\left(f(\theta)-G \frac{\cosh \theta}{\sinh ^{2} \theta}\right)+\frac{G}{4} \varphi(0) .
$$

\section{B Properties of the four-particle form factor}

In this appendix we consider the behaviour of the function

$$
F_{4}^{O}\left(\theta_{1}+i \pi,-\theta_{1}+i \pi,-\theta_{2}, \theta_{2}\right)
$$

near $\theta_{1}, \theta_{2}=0$. It follows from the form factor axioms that for infinitesimal $\theta_{1,2}$ the function above is antisymmetric in both variables and disappears whenever one of the rapidities goes to zero whith the other one being kept fixed. Therefore the only allowed singularity is of the form

$$
\frac{1}{\theta_{1} \theta_{2}}
$$

However, it is easy to see that this pole does not appear. Setting

$$
\theta_{1}=\theta+\varepsilon, \quad \theta_{2}=\theta
$$

and taking $\varepsilon \rightarrow 0$ results in [11]

$$
\lim _{\varepsilon \rightarrow 0} F_{4}^{O}(\theta+\varepsilon+i \pi,-\theta-\varepsilon+i \pi,-\theta, \theta)=\left(F_{4 c}^{O}(\theta,-\theta)-2 \varphi(2 \theta) F_{2 c}^{O}\right),
$$


which is well-defined for every $\theta$. Therefore a pole of $\frac{1}{\theta_{1} \theta_{2}}$ cannot appear and it follows that

$$
\lim _{\theta_{1}, \theta_{2} \rightarrow 0} F_{4}^{O}\left(\theta_{1}+i \pi,-\theta_{1}+i \pi,-\theta_{2}, \theta_{2}\right)=\lim _{\theta \rightarrow 0}\left(F_{4 c}^{O}(\theta,-\theta)-2 \varphi(2 \theta) F_{2 c}^{O}\right)=0 .
$$

Moreover, the first term of its Taylor expansion is proportional to $\theta_{1} \theta_{2}$, as required in (3.37) to cancel the double pole of $K_{\alpha}(-\theta) K_{\beta}(\theta)$.

\section{Summary of our results}

In this appendix we collected the results of section 3. The vacuum expectation value is given by

$$
\langle O(x)\rangle_{R}^{\alpha \beta}=\sum_{i, j} D_{i j}
$$

We calculated the terms with $i+j \leq 4$. The relative magnitudes of the individual contributions depend on $x$. However, a consistent ordering can be achieved by putting $x=R / 2$. This way $D_{i j}$ behaves in the large $R$ limit as $\mathcal{O}\left(e^{-(i+j) m R / 2}\right)$. A pictorial representation of the individual contributions can be found in figures 6 and 7 .

Terms of order $e^{-m R / 2}$

$$
\begin{aligned}
& D_{10}=\frac{g_{\alpha}}{2} F_{1}^{O} e^{-m x} \\
& D_{01}=\frac{g_{\beta}}{2} F_{1}^{O} e^{-m(R-x)}
\end{aligned}
$$

Terms of order $e^{-m R}$

$$
\begin{aligned}
D_{20} & =\frac{1}{2} \int \frac{d \theta}{2 \pi} K_{\alpha}(\theta) F_{2}^{O}(-\theta, \theta) e^{-2 m \cosh \theta x} \\
D_{02} & =\frac{1}{2} \int \frac{d \theta}{2 \pi} K_{\beta}(\theta) F_{2}^{O}(-\theta, \theta) e^{-2 m \cosh \theta(R-x)} \\
D_{11} & =\frac{g_{\alpha} g_{\beta}}{4} F_{2}^{O}(i \pi, 0) e^{-m R}
\end{aligned}
$$

Terms of order $e^{-3 m R / 2}$

$$
\begin{aligned}
D_{30}= & \frac{1}{2} \int \frac{d \theta}{2 \pi} K_{\alpha}(\theta) \frac{g_{\alpha}}{2} F_{3}^{O}(-\theta, \theta, 0) e^{-m(2 \cosh \theta+1) x} \\
D_{03}= & \frac{1}{2} \int \frac{d \theta}{2 \pi} K_{\beta}(\theta) \frac{g_{\beta}}{2} F_{3}^{O}(-\theta, \theta, 0) e^{-m(2 \cosh \theta+1)(R-x)} \\
D_{21}= & \frac{g_{\beta}}{4} \int \frac{d \theta}{2 \pi}\left(F_{3}^{O}(-\theta+i \pi, \theta+i \pi, 0) K_{\alpha}(\theta) e^{-2 m \cosh \theta x-m(R-x)}-\frac{2\left(g_{\alpha}\right)^{2} F_{1}^{O} \cosh \theta}{\sinh ^{2} \theta} e^{-m(R+x)}\right) \\
& +e^{-m(x+R)} g_{\beta}\left(g_{\alpha}\right)^{2} F_{1}^{O} \frac{\varphi(0)}{4} \\
D_{12}= & \frac{g_{\alpha}}{4} \int \frac{d \theta}{2 \pi}\left(F_{3}^{O}(i \pi,-\theta, \theta) K_{\beta}(\theta) e^{-2 m \cosh \theta(R-x)-m x}-\frac{2\left(g_{\beta}\right)^{2} F_{1}^{O} \cosh \theta}{\sinh ^{2} \theta} e^{-m(2 R-x)}\right) \\
& +e^{-m(2 R-x)} g_{\alpha}\left(g_{\beta}\right)^{2} F_{1}^{O} \frac{\varphi(0)}{4}
\end{aligned}
$$


Terms of order $e^{-2 m R}$

$D_{40}=\frac{1}{8} \int \frac{d \theta_{1}}{2 \pi} \frac{d \theta_{2}}{2 \pi} K_{\alpha}\left(\theta_{1}\right) K_{\alpha}\left(\theta_{2}\right) F_{4}^{O}\left(-\theta_{1}, \theta_{1},-\theta_{2}, \theta_{2}\right) e^{-2 m\left(\cosh \theta_{1}+\cosh \theta_{2}\right) x}$

$D_{04}=\frac{1}{8} \int \frac{d \theta_{1}}{2 \pi} \frac{d \theta_{2}}{2 \pi} K_{\beta}\left(\theta_{1}\right) K_{\beta}\left(\theta_{2}\right) F_{4}^{O}\left(-\theta_{1}, \theta_{1},-\theta_{2}, \theta_{2}\right) e^{-2 m\left(\cosh \theta_{1}+\cosh \theta_{2}\right)(R-x)}$

$D_{31}=\frac{g_{\alpha} g_{\beta}}{8} e^{-m R} \int \frac{d \theta}{2 \pi} K_{\alpha}(\theta) F_{4}^{O}(-\theta+i \pi, \theta+i \pi, i \pi, 0) e^{-2 m \cosh \theta x}$

$D_{13}=\frac{g_{\alpha} g_{\beta}}{8} e^{-m R} \int \frac{d \theta}{2 \pi} K_{\beta}(\theta) F_{4}^{O}(-\theta+i \pi, \theta+i \pi, i \pi, 0) e^{-2 m \cosh \theta(R-x)}$

$D_{22}=I_{22}+J_{22}$, where

$I_{22}=\frac{1}{4} \int \frac{d \theta_{1}}{2 \pi} \int \frac{d \theta_{2}}{2 \pi} K_{\alpha}\left(\theta_{1}\right) K_{\beta}\left(\theta_{2}\right) F_{4}^{O}\left(-\theta_{1}+i \pi, \theta_{1}+i \pi,-\theta_{2}, \theta_{2}\right) e^{-2 m \cosh \theta_{1} x-2 m \cosh \theta_{2}(R-x)}$

and

$J_{22}=F_{2}^{O}(i \pi, 0)\left\{\int \frac{d \theta}{2 \pi}\left(K_{\alpha}(-\theta) K_{\beta}(\theta) e^{-2 m \cosh \theta R}-\frac{\left(g_{\alpha} g_{\beta}\right)^{2} \cosh \theta}{4 \sinh ^{2} \theta} e^{-2 m R}\right)+\frac{\left(g_{\alpha} g_{\beta}\right)^{2}}{8} e^{-2 m R} \varphi(0)\right\}$ 

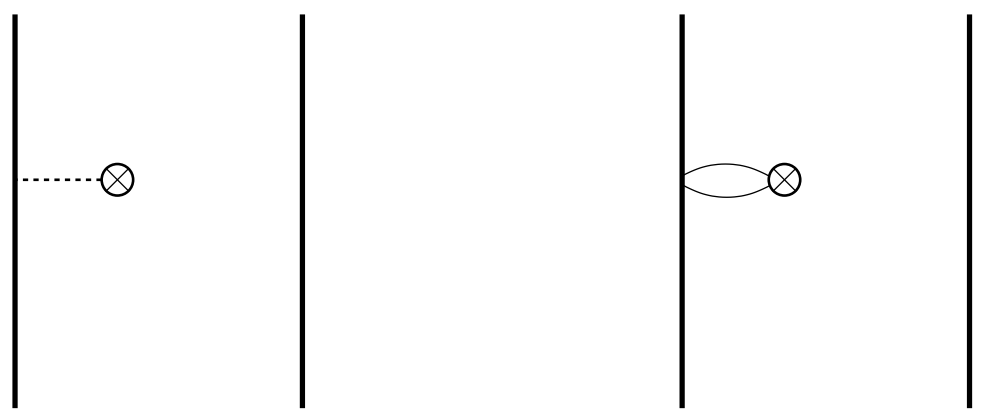

(a) $D_{10}$

(b) $D_{20}$
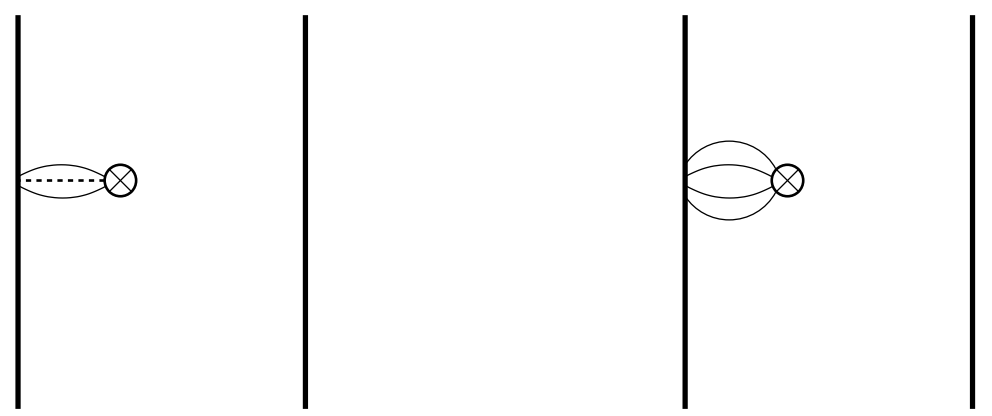

(c) $D_{30}$

(d) $D_{40}$
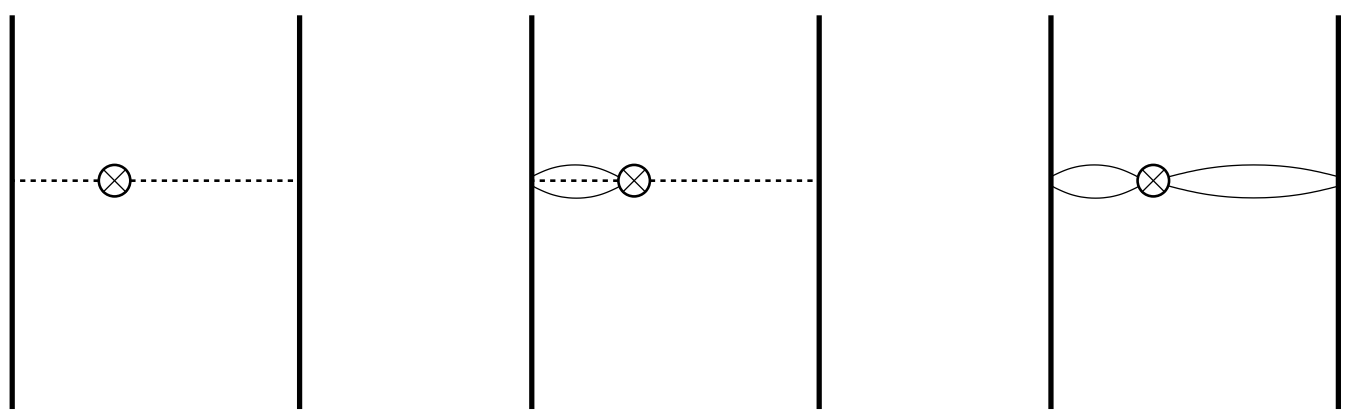

(e) $D_{11}$

(f) $D_{31}$

(g) $I_{22}$

Figure 6. Diagrams representing the different contributions to the v.e.v. Solid lines correspond to pairs of particles with opposite momenta. Dashed lines correspond to the propagation of zeromomentum particles. The diagrams depicting the terms $D_{i j}$ with $j>i$ can be obtained by switching the roles of the two boundaries. 


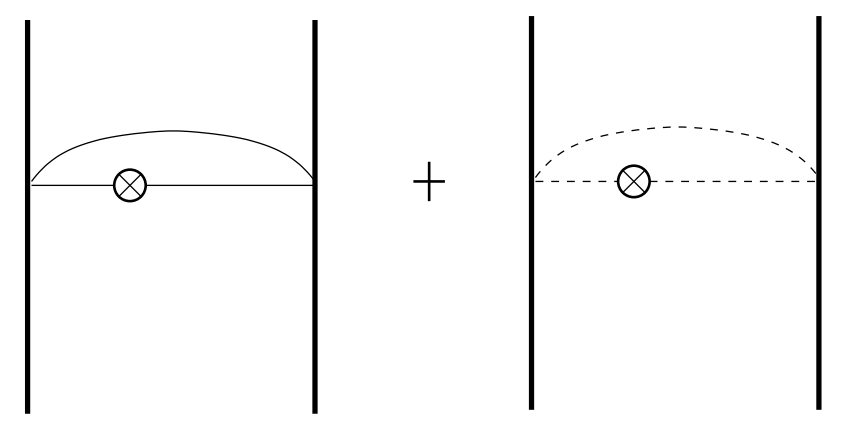

(a) $J_{22}$

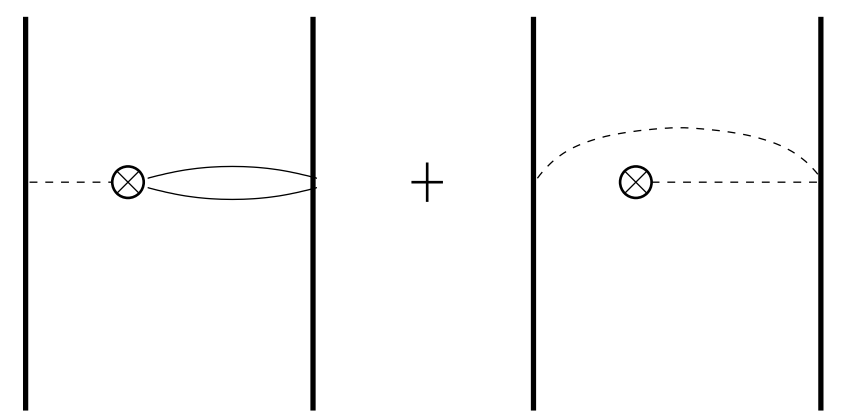

(b) $D_{12}$

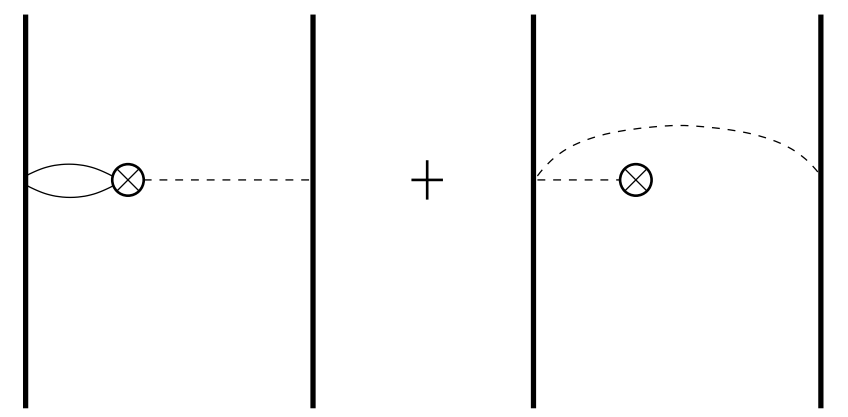

(c) $D_{21}$

Figure 7. Diagrams representing contributions with singular pieces. Solid lines correspond to pairs of particles with opposite momentum. Dashed lines correspond to the propagation of zeromomentum particles. 
Open Access. This article is distributed under the terms of the Creative Commons Attribution Noncommercial License which permits any noncommercial use, distribution, and reproduction in any medium, provided the original author(s) and source are credited.

\section{References}

[1] P. Calabrese and J.L. Cardy, Time-dependence of correlation functions following a quantum quench, Phys. Rev. Lett. 96 (2006) 136801 [cond-mat/0601225] [SPIRES].

[2] V. Gritsev, E. Demler, M. Lukin and A. Polkovnikov, Analysis of quench dynamics of coupled one dimensional condensates using quantum sine Gordon model, cond-mat/0702343 [SPIRES].

[3] D. Fioretto and G. Mussardo, Quantum Quenches in Integrable Field Theories, arXiv: 0911.3345 [SPIRES].

[4] A. Leclair, F. Lesage, S. Sachdev and H. Saleur, Finite temperature correlations in the one-dimensional quantum Ising model, Nucl. Phys. B 482 (1996) 579 [cond-mat/9606104] [SPIRES].

[5] R. Konik, A. LeClair and G. Mussardo, On Ising correlation functions with boundary magnetic field, Int. J. Mod. Phys. A 11 (1996) 2765 [hep-th/9508099] [SPIRES].

[6] D. Schuricht and F.H.L. Essler, Dynamical response functions in the quantum ising chain with a boundary, J. Stat. Mech. (2007) P11004 [arXiv:0709.1809].

[7] A. Leclair and G. Mussardo, Finite temperature correlation functions in integrable QFT, Nucl. Phys. B 552 (1999) 624 [hep-th/9902075] [SPIRES].

[8] O.A. Castro-Alvaredo and A. Fring, Finite temperature correlation functions from form factors, Nucl. Phys. B 636 (2002) 611 [hep-th/0203130] [SPIRES].

[9] H. Saleur, A comment on finite temperature correlations in integrable QFT, Nucl. Phys. B 567 (2000) 602 [hep-th/9909019] [SPIRES].

[10] G. Mussardo, On the finite temperature formalism in integrable quantum field theories, J. Phys. A 34 (2001) 7399 [hep-th/0103214] [SPIRES].

[11] B. Pozsgay and G. Takács, Form factors in finite volume II: disconnected terms and finite temperature correlators, Nucl. Phys. B 788 (2008) 209 [arXiv:0706.3605] [SPIRES].

[12] G. Takács, Finite temperature expectation values of boundary operators, Nucl. Phys. B 805 (2008) 391 [arXiv:0804.4096] [SPIRES].

[13] P. Dorey, M. Pillin, R. Tateo and G.M.T. Watts, One-point functions in perturbed boundary conformal field theories, Nucl. Phys. B 594 (2001) 625 [hep-th/0007077] [SPIRES].

[14] L. Samaj and Z. Bajnok, Exactly solvable model of the $2 D$ electrical double layer, Phys. Rev. E 72 (2005) 061503 [cond-mat/0508410] [SPIRES].

[15] B. Pozsgay and G. Takács, Form factors in finite volume I: form factor bootstrap and truncated conformal space, Nucl. Phys. B 788 (2008) 167 [arXiv:0706.1445] [SPIRES].

[16] F.H.L. Essler and R.M. Konik, Finite-temperature lineshapes in gapped quantum spin chains, Phys. Rev. B 78 (2008) 100403 [arXiv:0711.2524] [SPIRES].

[17] F.H.L. Essler and R.M. Konik, Finite temperature dynamical correlations in massive integrable quantum field theories, J. Stat. Mech. (2009) P09018 [arXiv:0907.0779]. 
[18] S. Ghoshal and A.B. Zamolodchikov, Boundary $S$ matrix and boundary state in two-dimensional integrable quantum field theory, Int. J. Mod. Phys. A 9 (1994) 3841 [hep-th/9306002] [SPIRES].

[19] Z. Bajnok, L. Palla and G. Takács, Finite size effects in quantum field theories with boundary from scattering data, Nucl. Phys. B 716 (2005) 519 [hep-th/0412192] [SPIRES].

[20] Z. Bajnok, L. Palla and G. Takács, Boundary one-point function, Casimir energy and boundary state formalism in D 1 dimensional QFT, Nucl. Phys. B 772 (2007) 290 [hep-th/0611176] [SPIRES].

[21] F.A. Smirnov, Form-factors in completely integrable models of quantum field theory, Adv. Ser. Math. Phys. 14 (1992) 1 [SPIRES].

[22] B. Berg, M. Karowski and P. Weisz, Construction of Green Functions from an Exact $S$ Matrix, Phys. Rev. D 19 (1979) 2477 [SPIRES].

[23] A.B. Zamolodchikov, Two point correlation function in scaling Lee-Yang model, Nucl. Phys. B 348 (1991) 619 [SPIRES].

[24] G. Delfino and G. Mussardo, The Spin spin correlation function in the two-dimensional Ising model in a magnetic field at $T=T-c$, Nucl. Phys. B 455 (1995) 724 [hep-th/9507010] [SPIRES].

[25] G. Delfino and P. Simonetti, Correlation Functions in the Two-dimensional Ising Model in a Magnetic Field at $T=T_{c}$, Phys. Lett. B 383 (1996) 450 [hep-th/9605065] [SPIRES].

[26] I. Affleck and A.W.W. Ludwig, Universal noninteger "ground state degeneracy" in critical quantum systems, Phys. Rev. Lett. 67 (1991) 161 [SPIRES].

[27] P. Dorey, D. Fioravanti, C. Rim and R. Tateo, Integrable quantum field theory with boundaries: The exact g-function, Nucl. Phys. B 696 (2004) 445 [hep-th/0404014] [SPIRES].

[28] P. Dorey, C. Rim and R. Tateo, Exact g-function flow between conformal field theories, arXiv:0911.4969 [SPIRES].

[29] M. Lüscher, Volume Dependence of the Energy Spectrum in Massive Quantum Field Theories. 1. Stable Particle States, Commun. Math. Phys. 104 (1986) 177 [SPIRES].

[30] T.R. Klassen and E. Melzer, On the relation between scattering amplitudes and finite size mass corrections in QFT, Nucl. Phys. B 362 (1991) 329 [SPIRES].

[31] Z. Bajnok and R.A. Janik, Four-loop perturbative Konishi from strings and finite size effects for multiparticle states, Nucl. Phys. B 807 (2009) 625 [arXiv:0807.0399] [SPIRES].

[32] Y. Hatsuda and R. Suzuki, Finite-Size Effects for Multi-Magnon States, JHEP 09 (2008) 025 [arXiv:0807.0643] [SPIRES].

[33] B. Pozsgay, Lüscher's mu-term and finite volume bootstrap principle for scattering states and form factors, Nucl. Phys. B 802 (2008) 435 [arXiv:0803.4445] [SPIRES].

[34] A. LeClair, G. Mussardo, H. Saleur and S. Skorik, Boundary energy and boundary states in integrable quantum field theories, Nucl. Phys. B 453 (1995) 581 [hep-th/9503227] [SPIRES].

[35] P. Dorey, A. Pocklington, R. Tateo and G. Watts, TBA and TCSA with boundaries and excited states, Nucl. Phys. B 525 (1998) 641 [hep-th/9712197] [SPIRES].

[36] Z. Bajnok, C. Rim and A. Zamolodchikov, Sinh-Gordon Boundary TBA and Boundary Liouville Reflection Amplitude, Nucl. Phys. B 796 (2008) 622 [arXiv:0710.4789] [SPIRES].

[37] G. Takács, Form factor perturbation theory from finite volume, Nucl. Phys. B 825 (2010) 466 [arXiv:0907.2109] [SPIRES]. 
[38] B. Pozsgay, work in progress.

[39] J.L. Cardy, Conformal invariance and the Yang-Lee edge singularity in two-dimensions, Phys. Rev. Lett. 54 (1985) 1354 [SPIRES].

[40] J.L. Cardy, Boundary Conditions, Fusion Rules and the Verlinde Formula, Nucl. Phys. B 324 (1989) 581 [SPIRES].

[41] J.L. Cardy and D.C. Lewellen, Bulk and boundary operators in conformal field theory, Phys. Lett. B 259 (1991) 274 [SPIRES].

[42] D.C. Lewellen, Sewing constraints for conformal field theories on surfaces with boundaries, Nucl. Phys. B 372 (1992) 654 [SPIRES].

[43] I. Runkel, Boundary structure constants for the A-series Virasoro minimal models, Nucl. Phys. B 549 (1999) 563 [hep-th/9811178] [SPIRES].

[44] J.L. Cardy and G. Mussardo, S Matrix of the Yang-Lee Edge Singularity in Two-Dimensions, Phys. Lett. B 225 (1989) 275 [SPIRES].

[45] A.B. Zamolodchikov, Thermodynamic Bethe ansatz in relativistic models. scaling three state Potts and Lee-Yang models, Nucl. Phys. B 342 (1990) 695 [SPIRES].

[46] A.B. Zamolodchikov, Mass scale in the sine-Gordon model and its reductions, Int. J. Mod. Phys. A 10 (1995) 1125 [SPIRES].

[47] F.A. Smirnov, The perturbated $C<1$ conformal field theories as reductions of sine-Gordon model, Int. J. Mod. Phys. A 4 (1989) 4213 [SPIRES].

[48] F.A. Smirnov, Reductions of the sine-Gordon model as a perturbation of minimal models of conformal field theory, Nucl. Phys. B 337 (1990) 156 [SPIRES].

[49] A.B. Zamolodchikov, Two point correlation function in scaling Lee-Yang model, Nucl. Phys. B 348 (1991) 619 [SPIRES].

[50] V. Fateev, S.L. Lukyanov, A.B. Zamolodchikov and A.B. Zamolodchikov, Expectation values of local fields in Bullough-Dodd model and integrable perturbed conformal field theories, Nucl. Phys. B 516 (1998) 652 [hep-th/9709034] [SPIRES].

[51] P. Dorey, I. Runkel, R. Tateo and G. Watts, $g$-function flow in perturbed boundary conformal field theories, Nucl. Phys. B 578 (2000) 85 [hep-th/9909216] [SPIRES].

[52] V.P. Yurov and A.B. Zamolodchikov, Truncated conformal space approach to scaling Lee-Yang model, Int. J. Mod. Phys. A 5 (1990) 3221 [SPIRES].

[53] G. Feverati, K. Graham, P.A. Pearce, G.Z. Toth and G. Watts, A renormalisation group for TCSA, hep-th/0612203 [SPIRES].

[54] M. Kormos, I. Runkel and G.M.T. Watts, Defect flows in minimal models, JHEP 11 (2009) 057 [arXiv:0907.1497] [SPIRES].

[55] I. Runkel, Boundary Problems in Conformal Field Theory, Ph.D Thesis (2000) http://www.mth.kcl.ac.uk/staff/i_runkel/PDF/phd.pdf.

[56] F.H.L. Essler and R.M. Konik, Applications of massive integrable quantum field theories to problems in condensed matter physics, cond-mat/0412421 [SPIRES].

[57] G. Delfino, G. Mussardo and P. Simonetti, Correlation functions along a massless flow, Phys. Rev. D 51 (1995) 6620 [hep-th/9410117] [SPIRES].

[58] D.C. Cabra, A. Honecker, G. Mussardo and P. Pujol, A non-perturbative approach to the random-bond Ising model, J. Phys. A 30 (1997) 8415 [hep-th/9705180] [SPIRES]. 